\title{
¿Contribution of Ocean Physics and Dynamics at Different Scales to Heat Uptake in Low-Resolution AOGCMs
}

\author{
Oleg A. SAenko, ${ }^{a}$ Jonathan M. Gregory, ${ }^{\mathrm{b}, \mathrm{c}}$ StePhen M. Griffies, ${ }^{\mathrm{d}, \mathrm{e}}$ MatTHew P. Couldrey, ${ }^{\mathrm{b}}$ \\ AND FABIO BOEIRA DIAS ${ }^{\mathrm{f}}$

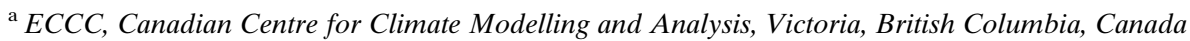 \\ ${ }^{\mathrm{b}}$ NCAS, University of Reading, Reading, United Kingdom \\ ${ }^{\mathrm{c}}$ Met Office Hadley Centre, Exeter, United Kingdom \\ ${ }^{\mathrm{d}}$ NOAA/Geophysical Fluid Dynamics Laboratory, Princeton, New Jersey \\ ${ }^{\mathrm{e}}$ Princeton University Atmospheric and Oceanic Sciences Program, Princeton, New Jersey \\ ${ }_{\mathrm{f}}^{\mathrm{f}}$ INAR, University of Helsinki, Helsinki, Finland
}

(Manuscript received 17 August 2020, in final form 9 November 2020)

\begin{abstract}
Using an ensemble of atmosphere-ocean general circulation models (AOGCMs) in an idealized climate change experiment, this study quantifies the contributions to ocean heat uptake (OHU) from ocean physical parameterizations and resolved dynamical processes operating at different scales. Analysis of heat budget diagnostics reveals a leadingorder global heat balance in the subsurface upper ocean in a steady state between the large-scale circulation warming it and mesoscale processes cooling it, and shows that there are positive contributions from processes on all scales to the subsurface OHU during climate change. There is better agreement among the AOGCMs in the net OHU than in the individual scales/ processes contributing to it. In the upper ocean and at high latitudes, OHU is dominated by small-scale diapycnal processes. Below $400 \mathrm{~m}$, OHU is dominated by the superresidual transport, representing large-scale ocean dynamics combined with all parameterized mesoscale and submesoscale eddy effects. Weakening of the AMOC leads to less heat convergence in the subpolar North Atlantic and less heat divergence at lower latitudes, with a small overall effect on the net Atlantic heat content. At low latitudes, the dominance of advective heat redistribution is contrary to the diffusive OHU mechanism assumed by the commonly used upwelling-diffusion model. Using a density water-mass framework, it is found that most of the OHU occurs along isopycnal directions. This feature of $\mathrm{OHU}$ is used to accurately reconstruct the global vertical ocean warming profile from the surface heat flux anomalies, supporting advective (rather than diffusive) models of OHU and sea level rise.
\end{abstract}

KEYWORDS: Climate change; Heat budgets/fluxes; Ocean dynamics; Isopycnal mixing

\section{Introduction}

Among the major components of the Earth system (ocean, land, ice, and atmosphere), the ocean by far dominates the uptake of heat associated with anthropogenic greenhouse gas emissions (e.g., Otto et al. 2013). Once in the ocean, heat anomalies are transported by a variety of processes that allow heat to penetrate into the ocean interior well beneath the surface boundary (e.g., Levitus et al. 2012). This ocean heat uptake (OHU) moderates surface atmospheric climate warming and, through thermal expansion of seawater and melting of ice shelves (with associated increased land-ice melt), contributes to global and regional sea level rise (e.g., Church et al. 2013). Observation-based reconstructions (e.g., Zanna et al. 2019) and climate change simulations based on atmosphere-ocean general circulation models (AOGCMs) (e.g., Gregory 2000; Kuhlbrodt et al. 2015; Exarchou et al. 2015) indicate that $\mathrm{OHU}$ is highly nonuniform in space, which in turn contributes to regional changes in dynamic sea level. Projected magnitudes of dynamic sea level change can be comparable to global-mean sea level rise due to thermal expansion

¿ Denotes content that is immediately available upon publication as open access.

Corresponding author: Oleg Saenko,oleg.saenko@canada.ca (e.g., Yin et al. 2010; Gregory et al. 2016). Therefore, improved understanding of $\mathrm{OHU}$, including its vertical and horizontal structure and its spread among AOGCMs, is essential for improved projections of surface climate and sea level changes.

Several previous studies performed process-based analyses of OHU in climate change experiments, typically based on either one or a few AOGCMs (Gregory 2000; Kuhlbrodt et al. 2015; Exarchou et al. 2015), or on idealized-basin models (Saenko 2006; Morrison et al. 2013). Among other findings, these studies highlighted the importance of different physical processes for OHU in different regions. In particular, for high-latitude regions with weak vertical stratification, OHU was found to be dominated by changes in the processes affecting ventilation, such as vertical convective mixing and parameterized mesoscale eddy-induced effects. In low-latitude regions, changes in ocean heat content (OHC) were dominated by changes in large-scale heat advection. These findings are broadly supported by our analysis using a more thorough suite of models. In particular, we find that the main effect from diapycnal mixing processes is to make the subsurface North Atlantic and Southern Ocean warmer, while the combined effect from all other processes is to make the subpolar Atlantic colder and most of the rest of the ocean warmer (Fig. 1, with a more detailed discussion provided in section 3).

Despite considerable progress in understanding the contribution of individual processes to OHU in AOGCMs, many questions remain. In particular, the substantial spread among 
(a) Net OHC change below $200 \mathrm{~m}$

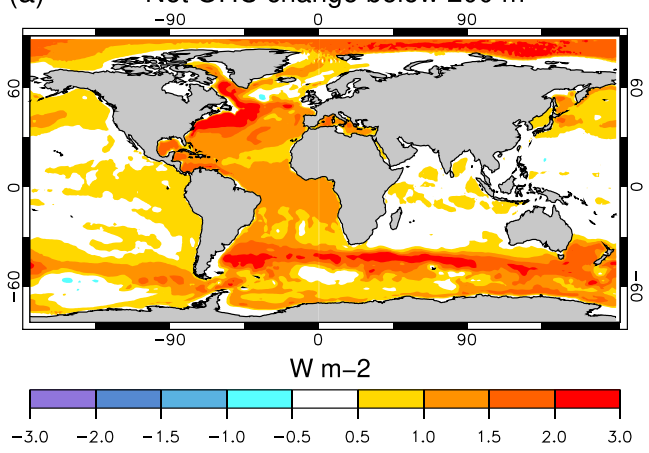

(c) $\mathrm{OHC}$ change due to super-residual transport

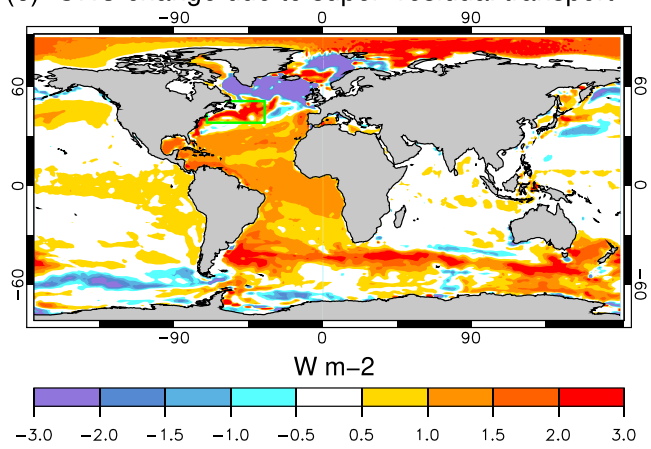

(b) $\mathrm{OHC}$ change due to diapycnal mixing

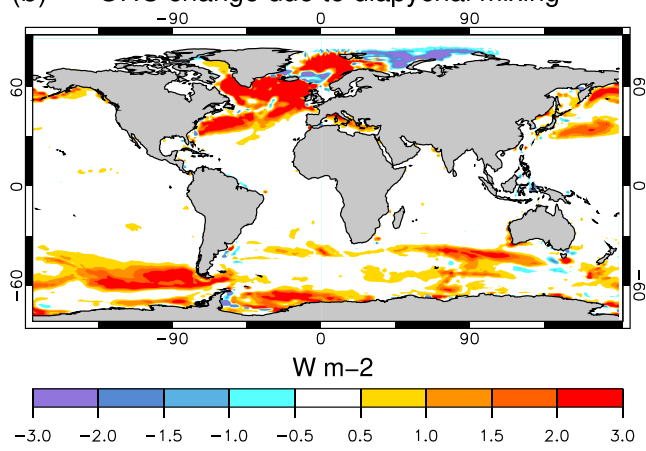

(d) Standard deviation of the net $\mathrm{OHC}$ change

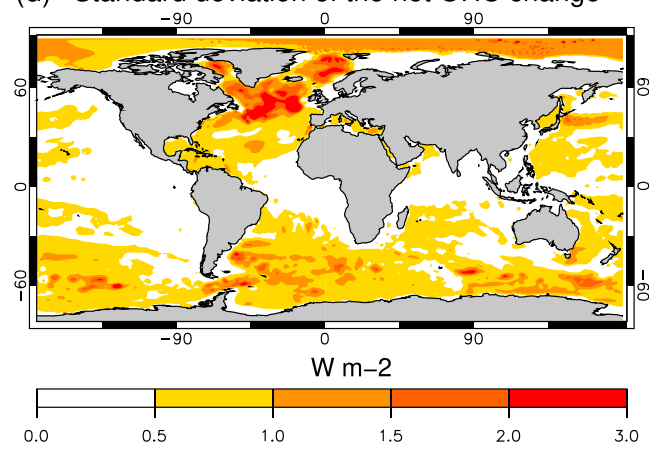

FIG. 1. Model-mean rate of OHC change below $200 \mathrm{~m}$ (1pctCO2 wrt piControl) over the first 70 years: (a) net $\mathrm{OHC}$ change due to all processes and its partitioning into contributions from (b) all forms of the diapycnal mixing in the analyzed AOGCMs and (c) the superresidual transport that combined the large-scale heat advection with all eddy heat transport processes (see text for details). Positive values correspond to heat being added to the region deeper than $200 \mathrm{~m}$, whereas a negative number sees cooling below $200 \mathrm{~m}$. The color scale is limited to $\pm 3 \mathrm{~W} \mathrm{~m}^{-2}$ for plotting purposes. (d) Ensemble STD of the net OHC change shown in (a). The green box in (c) is the Gulf Stream region to which we refer in section $3 \mathrm{a}(3)$.

AOGCMs in terms of the processes regulating OHU (Exarchou et al. 2015), both parameterized and resolved, needs to be better understood. Here, we further elaborate on these processes by building on earlier studies by Gregory (2000), Kuhlbrodt et al. (2015), and Exarchou et al. (2015). Specifically, using a larger suite of AOGCMs, we focus on contributions to OHU arising from both parameterized and resolved ocean physical processes that operate at different space/time scales; namely, resolved large-scale circulation along with parameterized mesoscale and submesoscale eddy motions as well as small-scale turbulent mixing. We also present the associated uncertainties and show that, separately for individual scales, the uncertainties are larger than the uncertainty in the net global OHU. We apply two frameworks for our heat budget analysis: a traditional framework working in native model grid space and involving horizontal and vertical integration of heat budget equations, and a density space water-mass framework described in the next section.

\section{Model diagnostics and analysis frameworks}

In this section we describe the model diagnostics used for the heat budget and outline the analysis frameworks.

\section{a. Models, experiments, and diagnostics}

We analyze model output from a climate change experiment where atmospheric $\mathrm{CO}_{2}$ concentration increases at $1 \% \mathrm{yr}^{-1}$ (1pctCO2), along with the corresponding output from a preindustrial control experiment (piControl). In what follows, unless stated otherwise, all heat budget terms represent changes (1pctCO2 with respect to piControl), averaged over the first 70 years; that is, until atmospheric $\mathrm{CO}_{2}$ has doubled. The analyzed AOGCMs, all having a nominal ocean resolution of about $1^{\circ}$, are listed in Table 1 and information on the heat budget diagnostics we analyze is provided in Table 2. A detailed explanation of the heat budget terms in Table 2 is given in Griffies et al. (2016; section 9) (see also Gregory et al. 2016; section 2f). Briefly, these diagnostics are as follows:

temprmadvect contains heat convergence from all forms of advection, both resolved and parameterized eddy induced;

temppadvect contains heat convergence from parameterized mesoscale eddy-induced advection (e.g., Gent et al. 1995) and parameterized submesoscale eddy-induced advection (e.g., Fox-Kemper et al. 2011; not all models include this latter term in their simulations); 
TABLE 1. Information on the AOGCMs analyzed in this study. Ocean grid spacing (Res.) is indicated approximately; it varies in some AOGCMs. The choices for representation of mesoscale eddy advection (Meso. adv.) and diffusion (Meso. dif.) follow either the formulations in Gent and McWilliams (1990, GM90) and Redi (1982, R82), or the skew flux formulation in Griffies (1998, G98); $V$ and $F$ indicate if the corresponding eddy transfer coefficients are variable in space and time or fixed; the ranges or values of these coefficients (in $\mathrm{m}^{2} \mathrm{~s}^{-1}$ ) are also indicated, if known. Some models include the Fox-Kemper et al. (2011) parameterization of mixed layer eddies (Submeso.). Marked with* are the AOGCMs for which the heat tendency diagnostics (Table 2) were available as monthly averages and were used in the heat budget analysis in density space discussed in section $3 b$; for all other models these diagnostics were available only as annual averages.

\begin{tabular}{rlcllll}
\hline \hline \multicolumn{1}{c}{ AOGCM } & Res. (lat $\times$ lon; levels) & \multicolumn{1}{c}{ Meso. adv. } & \multicolumn{1}{c}{ Meso. dif. } & Submeso. & \multicolumn{1}{c}{ Reference } \\
\hline 1 & ACCESS-CM2* & $1.0^{\circ} \times 1.0^{\circ} ; 50$ & $\mathrm{G} 98, V, 100-1200$ & $\mathrm{G} 98, F, 300$ & Yes & Bi et al. (2020) \\
2 & CanESM2 & $1.0^{\circ} \times 1.4^{\circ} ; 40$ & $\mathrm{GM} 90, V, 100-2000$ & $\mathrm{R} 82, F, 1000$ & No & Yang and Saenko (2012) \\
3 & CanESM5 & $1.0^{\circ} \times 1.0^{\circ} ; 45$ & $\mathrm{GM} 90, V, 100-2000$ & $\mathrm{R} 82, V,<1000$ & No & Swart et al. (2019) \\
4 & CESM2 & $1.0^{\circ} \times 1.0^{\circ} ; 60$ & $\mathrm{G} 98, V, 300-3000$ & $\mathrm{G} 98, V, 300-3000$ & Yes & Danabasoglu et al. (2012) \\
5 & GFDL-ESM2M* & $1.0^{\circ} \times 1.0^{\circ} ; 50$ & $\mathrm{G} 98, V, 100-800$ & $\mathrm{G} 98, F, 600$ & Yes & Dunne et al. (2012) \\
6 & HadCM3* & $1.2^{\circ} \times 1.2^{\circ} ; 20$ & $\mathrm{GM} 90, V, 350-2000$ & $\mathrm{G} 98, F, 1000$ & No & Gordon et al. (2000) \\
7 & HadGEM2-ES* & $1.0^{\circ} \times 1.0^{\circ} ; 40$ & $\mathrm{G} 98, V, \geq 150$ & $\mathrm{G} 98, F, 500$ & No & Johns et al. (2006) \\
8 & HadGEM3-GC31-LL* & $1.0^{\circ} \times 1.0^{\circ} ; 75$ & $\mathrm{GM} 90, V, \leq 1000$ & $\mathrm{R} 82, F, 1000$ & No & Kuhlbrodt et al. $(2018)$ \\
9 & IPSL-CM6A-LR & $1.0^{\circ} \times 1.0^{\circ} ; 75$ & $\mathrm{GM} 90, V$, No info & $\mathrm{G} 98, V, \leq 1000$ & Yes & Boucher et al. (2020) \\
10 & MPI-ESM1.2-LR & $1.0^{\circ} \times 1.4^{\circ} ; 40$ & $\mathrm{G} 98, V, \leq 250$ & $\mathrm{G} 98, V, \leq 1000$ & No & Gutjahr et al. (2019) \\
11 & MRI-ESM2.0 & $0.5^{\circ} \times 1.0^{\circ} ; 61$ & $\mathrm{GM90,V,300-1500}$ & $\mathrm{R} 82, F, 1500$ & No & Yukimoto et al. (2019) \\
\hline
\end{tabular}

temppsmadvect contains heat convergence from parameterized submesoscale eddy-induced advection alone (for those models that include this term; see Table 1);

temppmdiff represents heat convergence from parameterized diffusive fluxes associated with transient mesoscale eddies [i.e., isopycnal diffusion as in Redi (1982) and Griffies et al. (1998)];

tempdiff contains heat convergence from parameterized diapycnal processes including vertical convective adjustment.

The choices for mesoscale eddy-induced advection and isopycnal diffusion, along with the constraints on the associated eddy transfer coefficients made by each of the models, are presented in Table 1.

TABLE 2. Heat budget terms $\left(\mathrm{W} \mathrm{m}^{-2}\right)$ analyzed in this study. A detailed explanation is provided in Griffies et al. (2016), where terms 1-6 are prefixed by "opot" or "ocon" for, respectively, potential or conservative temperature. Note that term 2 includes term 3 , and term 3 includes term 4. Term 7 "other" represents the combined effect from the processes not included in terms 1-6 [see Griffies et al. (2016) for examples], inferred by taking the difference between the net tendency (term 1) and the sum of residual mean advection (term 2), mesoscale diffusion (term 5) and diapycnal mixing (term 6).

\begin{tabular}{lll}
\hline \hline & \multicolumn{1}{c}{ Name } & \multicolumn{1}{c}{ Heat budget terms } \\
\hline 1 & Temptend & Net temperature tendency \\
2 & Temprmadvect & Residual mean advection \\
3 & Temppadvect & Net eddy-induced advection \\
4 & Temppsmadvect & Submesoscale eddy-induced advection \\
5 & Temppmdiff & Mesoscale diffusion \\
6 & Tempdiff & Diapycnal mixing \\
7 & Other & Remaining processes \\
\hline
\end{tabular}

\section{b. Partitioning the heat budget}

In the traditional framework, we focus on OHU below 200-m depth, thus excluding (in most regions) the upper layer of strong surface-intensified mixing and solar penetration. Therefore, the grid cell heat budget takes the following form (Griffies et al. 2016): ${ }^{1}$

temptend $=$ temprmadvect + temppmdiff + tempdiff + other .

The heat budget terms in Table 2 are grouped to reflect the physical and dynamical processes operating at different spatial scales. For this purpose, the net OHU (All scales), as given by the temptend term, is partitioned into the following contributions:

Large: large-scale ocean flows explicitly represented by the model's resolved velocity field;

Meso: parameterized ocean mesoscale eddy effects, both advective and diffusive, as well as parameterized submesoscale eddy-induced advection (if included in the model);

Small: parameterized processes associated with diapycnal mixing, such as gravitationally induced convection, boundary layer and shear-driven mixing, tidal mixing, as well as all remaining diapycnal effects (e.g., parameterized overflow-driven mixing).

In the adopted notations,

$$
\begin{aligned}
& \text { Large }=\text { temprmadvect }- \text { temppadvect }, \\
& \text { Meso }=\text { temppadvect }+ \text { temppmdiff },
\end{aligned}
$$

\footnotetext{
${ }^{1}$ There is a typo in Eqs. (L5) and (L6) in Griffies et al. (2016) where instead of opottempadvect there should be opottemprmadvect.
} 


$$
\begin{aligned}
\text { Small } & =\text { tempdiff }+ \text { other }, \text { and } \\
\text { All scales } & =\text { Large }+ \text { Meso }+ \text { Small } .
\end{aligned}
$$

In addition, we shall present the OHU associated with the superresidual transport (SRT) (Kuhlbrodt et al. 2015; Dias et al. 2020a), where SRT is defined as the sum

$$
\mathrm{SRT}=\text { Large }+ \text { Meso }
$$

The SRT is the contribution to OHU associated with the explicitly resolved advection combined with all forms of parameterized mesoscale and submesoscale eddy-induced advection and isopycnal diffusion. The SRT contribution to OHU (e.g., Fig. 1c) provides a direct link between ocean models that parameterize mesoscale eddy-induced advection and isopycnal diffusion (such as the models in the current study) and the growing suite of ocean and climate models that explicitly resolve rather than parameterize these eddy transport processes. Note that with the adopted notations,

$$
\text { All scales }=\text { SRT }+ \text { Small }
$$

We will also consider separately the OHU effect from all parameterized (in these AOGCMs) processes, Param $=$ Meso + Small, so that

$$
\text { All scales }=\text { Large }+ \text { Param } .
$$

This decomposition is aimed at estimating the combined contribution of all subgrid-scale processes to OHU in AOGCMs with low-resolution ocean components, along with the associated $\mathrm{OHU}$ uncertainties.

\section{c. Projection of the Eulerian heat budget onto density surfaces}

In addition to heat budget analysis involving horizontal and vertical integration of Eq. (5), in section 3b we employ a potential density space water-mass framework as first introduced using a temperature space framework by Walin (1982) and more recently by Holmes et al. (2019). Our analysis of an Eulerian heat budget projection onto density surfaces provides further insight on the OHU processes active in AOGCMs and, in particular, on the link between heat input to different density classes at the surface and vertical $\mathrm{OHU}$ profiles in the ocean interior. It also helps to clarify the role of heat advection by the residual mean velocity. Namely, in the potential density space framework, advective heat transport across isopycnals can naturally arise as an important (physical) component of the heat budget in the presence of mixing, while in the diathermal framework the role of temperature advection in the heat budget is not considered (Walin 1982; Holmes et al. 2019).

For our purposes of separating the role of ocean physics and dynamics at different scales, the applied projection of the Eulerian heat budget onto the position of potential density surfaces is as follows. Consider the whole ocean domain, so that Eq. (5) takes the form

$$
\text { All scales }=\text { Large }+ \text { Meso }+ \text { Small }+ \text { Flux } \delta(z-\eta),
$$

where we assume that there are no sources or sinks of heat other than due to the net heat flux (Flux) across the surface boundary, with $\delta(z-\eta)$ the Dirac delta function that enables us to incorporate surface boundary fluxes within the same formalism as interior processes [with $z=\eta(x, y, t)$ being the ocean free surface height]. Integrating Eq. (9) over all ocean regions with densities larger than any given density $\rho$ gives

$$
\begin{aligned}
\mathscr{H}(\rho, t)= & \iiint_{\rho^{\prime}(x, y, z, t) \geq \rho}(\text { Large }+ \text { Meso }+ \text { Small }) d V \\
& +\iint_{\rho^{\prime}(x, y, 0, t) \geq \rho} \text { Flux } d A,
\end{aligned}
$$

where $\mathscr{H}(\rho, t)=\iiint_{\rho^{\prime}(x, y, z, t) \geq \rho}$ (All scales) $d V$ represents the net heat convergence within all water classes denser than $\rho$, while the terms on the right side represent contributions from diapycnal heat transports associated with the three different scales as well as the surface transformation. Averaging in time, represented with overbar, gives

$$
\begin{aligned}
\overline{\mathscr{H}(\rho, t)} & =\overline{\iiint_{\rho^{\prime}(x, y, z, t) \geq \rho}(\text { Large }+ \text { Meso }+ \text { Small }) d V} \\
& +\overline{\iint_{\rho^{\prime}(x, y, 0, t) \geq \rho} \operatorname{Flux} d A .}
\end{aligned}
$$

We note that, because the time averaging is applied to $\iiint_{\rho^{\prime}(x, y, z, t) \geq \rho}() d$.$V , the term on the left side of Eq. (11) does$ not have to vanish even if the averaged in time Eulerian time derivative of ocean temperature does so locally [see Groeskamp et al. (2014) for a comprehensive discussion on the subject with insightful examples]. However, as we shall see in section $3 \mathrm{~b}$ (Fig. 9a), at a statistical steady state this term is, in general, small compared to the other terms (although nonnegligible). This implies that at a statistical steady state heat loss at the surface by water classes denser than $\rho$ is mostly resupplied by diapycnal heat transport at different scales in the ocean interior. The diapycnal transports can be associated with different physical and dynamical processes, including the heat advection across density surfaces that occurs in the presence of mixing.

When the simulated climate system is perturbed, such as in 1 pctCO2, $\mathscr{H}(\rho)$ departs from zero. In a special case when $\rho$ corresponds to the lightest water $\rho=\rho_{\text {lightest-water, Eq. (10) }}$ simplifies to

$$
\mathscr{H}\left(\rho_{\text {lightest-water }}\right)=\iint_{\rho^{\prime}(x, y, 0, t) \geq \rho_{\text {lightest-water }}} \operatorname{Flux} d A,
$$

which simply states that the net OHU is given by the net heat input at the surface (in the absence of other heat sources).

For the projection of the Eulerian heat budget onto the position of potential density surfaces in section $3 \mathrm{~b}$, in addition to the heat budget terms listed in Table 2, we also use surface heat flux with solar flux and ocean temperature and salinity (to compute density). In practice, the calculation involves binning the ocean into density classes, which is conceptually similar to the temperature binning procedure employed by Holmes et al. (2019) for their heat budget analysis. We use $0.1 \sigma_{\theta}$ bins, where $\sigma_{\theta}=\rho_{\theta}-1000 \mathrm{~kg} \mathrm{~m}^{-3}$, with $\rho_{\theta}$ being potential density referenced to 0 dbar. It was found that further decrease in the size of the density bins had little impact on the results and did not affect the conclusions. Ideally, such a calculation should be 


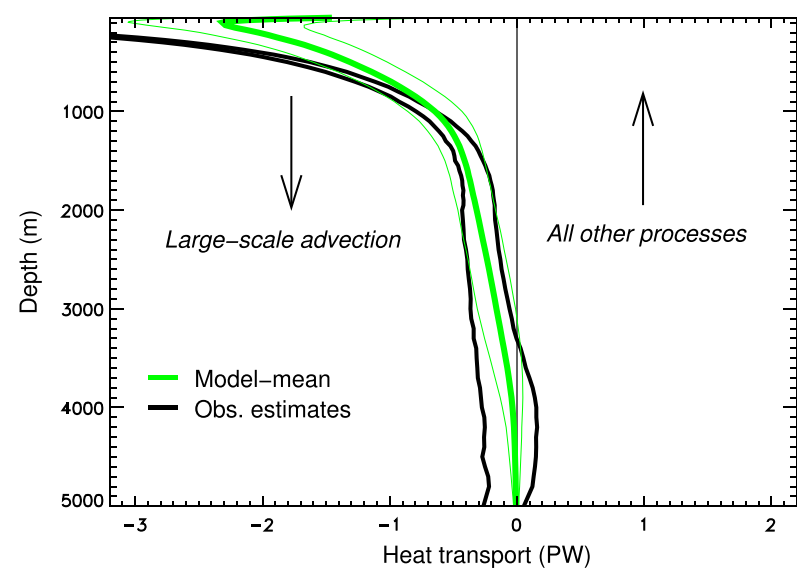

FIG. 2. Time-mean (70 years of piControl) and model-mean global vertical heat transport (PW) due to the explicitly simulated (by the analyzed AOGCMs) large-scale ocean circulation (green), with thin lines corresponding to \pm 1 intermodel STD. Also presented are two observational estimates (black) of heat transport associated with ocean circulation, which obeys the linear vorticity balance (Cummins et al. 2016). These two estimates are based on somewhat different assumptions about the reference meridional geostrophic velocity (see Cummins et al. 2016 for details). On the long-term mean, the downward heat transport due to the largescale advection is expected to be closely balanced by an equal and opposite (i.e., upward) transport associated with the combined effect from all other (parameterized in these AOGCMs) processes, as illustrated by arrows.

performed "online" while models are running. Online binning is needed to reduce inaccuracies associated with nonlinear effects. However, we did not have access to online diagnostics in the suite of models, so we instead did the calculation "offline," using monthly data. In selected tests, we found that in AOGCMs with relatively coarse-resolution oceans, such as analyzed in this study, using monthly data in this calculation leads to almost the same results as when using daily data. Models with all the required output available as monthly averages are marked with an asterisk in Table 1 . The results of the density space heat budget analysis presented in section $3 \mathrm{~b}$ represent model-mean and time-mean quantities corresponding to years $61-70$ of 1 pctCO 2 and piControl. More details on the calculation as well as on how it relates with the water-mass transformation (WMT) framework described in Groeskamp et al. (2019) are presented in appendix A.

\section{d. Comments on observational constraints}

Before proceeding with the OHU analysis, it is useful to understand how the simulated heat transports that correspond to ocean physics and dynamics operating at different scales compare against observational counterparts. Unfortunately, reliable observations of vertical heat fluxes are not available for the global ocean. However, indirect approaches can be used for estimating some of them. In particular, Cummins et al. (2016) present near-global observational estimates of the vertical heat transport associated with time-mean, large-scale motions. Cummins et al. (2016) obtained their vertical heat transports using climatological ocean temperature and the linear vorticity balance, $f w_{z}=\beta v$. The latter was used to estimate climatological vertical velocity $w$ in the ocean interior from climatological wind stress and density and from observational estimates of the meridional component of absolute geostrophic velocity $v$ at a reference depth.

Figure 2 compares the Cummins et al. (2016) observational estimates with the model-mean vertical heat transport due to Large. Overall, the simulated vertical heat transport is consistent with the observational estimates. However, there is a considerable spread among the AOGCMs even in this vertical heat flux that these models are expected to simulate explicitly. Discrepancies between the model-simulated and observationestimated heat transports are large in the upper several hundred meters, also noted in Cummins et al. (2016, their Fig. 6). These discrepancies could originate from model biases in either the large-scale temperature field or vertical velocity or both. While a detailed analysis of these discrepancies is beyond our scope here, we note that biases in simulated vertical velocity, particularly in the upper ocean, can be strongly affected by biases in wind stress curl simulated by AOGCMs. In the deep ocean, they provide two observational heat transports corresponding to somewhat different assumptions that are equally justified (see Cummins et al. 2016 for details). Unfortunately, these two estimates diverge. While this divergence complicates a comparison with the model-simulated transports, we note that the model-mean heat transport curve is positioned roughly in between the two observational curves in the deep ocean, with the model spread decreasing toward the abyssal ocean. Since our analysis of OHU is confined to the upper $2000 \mathrm{~m}$, difficulties with deep ocean heat transport are not directly relevant to our analysis.

Figure 2 also confirms a finding by Gregory (2000), and more recently confirmed by others (e.g., Griffies et al. 2015), that large-scale ocean circulation transports heat downward when horizontally averaged over the globe. This transport can also be understood based on energetic arguments (Gnanadesikan et al. 2005; Gregory and Tailleux 2011), suggesting that largescale wind-driven ocean circulation is expected to generate potential energy, via fluxing more buoyant waters downward and less buoyant upward on the global mean. A major contribution to this process comes from the Southern Ocean where Ekman pumping fluxes relatively warm (cold) waters downward (upward) roughly north (south) of $45^{\circ} \mathrm{S}$ (e.g., Gregory 2000; Cummins et al. 2016).

\section{e. A kinematic constraint on steady vertical heat transport}

In a steady state there is zero horizontal area integrated vertical heat convergence in the interior ocean

$$
\Rightarrow \int_{\text {global ocean }} \frac{\partial\left(w \rho C_{p} \Theta+J^{z}\right)}{\partial z} d x d y=0,
$$

where $w \rho C_{p} \Theta$ is the vertical advective flux of heat from the resolved model flow ( $w$ is vertical velocity, $\rho$ is ocean density, $C_{p}$ is heat capacity, and $\Theta$ is Conservative Temperature), and $J^{z}$ is the vertical component of the subgrid scale heat flux. We next 
(a) Rate of heating: piControl

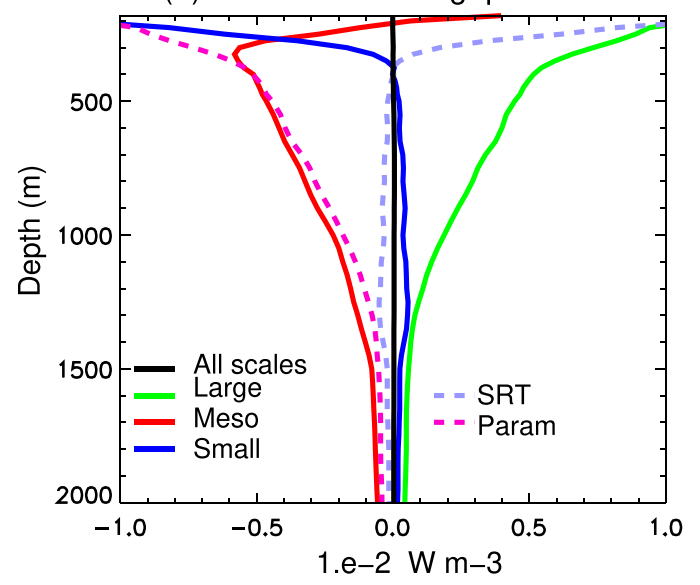

(b) Inter-model STD: piControl

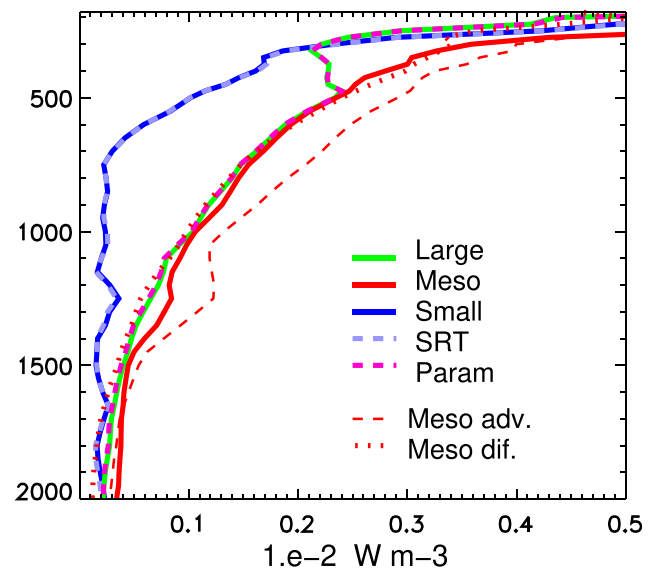

FIG. 3. (a) Global-mean and time-mean (70 years) profiles of heat convergences in piControl corresponding to the net heating rate (All scales) and its partitioning into contributions from the resolved circulation (Large), all mesoscale and submesoscale eddy-related processes (Meso) and all diapycnal and other effects (Small). Also presented separately are the contributions from the superresidual transport (SRT $=$ Large + Meso) and all parameterized (in these AOGCMs) processes (Param $=$ Small + Meso). (b) Profiles of intermodel STDs corresponding to the heat convergence profiles in (a); also presented are the STDs corresponding to heat convergence due to eddy advection (Meso adv.) and diffusion (Meso dif.).

observe that the ocean gains and loses heat predominantly through the sea surface, with negligible sources from viscous dissipation (i.e., joule heating) and only a small amount from bottom geothermal heating (order tens of milliwatts per square meter). If we disregard the latter as well, a vertical integral of Eq. (13) from the ocean bottom upward means that the steady, global horizontally integrated, vertical heat transport vanishes on any horizontal level below the influence of surface boundaries; that is,

steady, interior ocean, zero geothermal, zero joule

$$
\Rightarrow \int_{\text {global ocean }}\left(w \rho C_{p} \Theta+J^{z}\right) d x d y=0 .
$$

Consequently, if we partition vertical heat transport into any variety of terms, such as the separations described above, then the net vertical heat transport by all processes at any depth must sum to zero (as illustrated by arrows in Fig. 2). We make use of the constraint (14) as part of our analysis of vertical heat transport. Note that a similar constraint cannot be applied to meridional heat transport since it is strongly affected by surface fluxes at all latitudes.

\section{Results}

\section{a. Controls on the heat budget}

\section{1) VERTICAL HEAT CONVERGENCE AT STATISTICAL STEADY STATE}

We begin our analysis with a brief discussion of the heat budget in piControl for the model ensemble mean, focusing on the ocean between 200 and $2000 \mathrm{~m}$, which takes up most of the heat [we discuss heat uptake in 1 pctCO2 in section $3 \mathrm{a}(2)$ ]. In the global horizontal area mean, the heat convergence due to
Large warms the 200-2000-m layer (Fig. 3a), as implied by the corresponding transport (Fig. 2). The interior ocean heating by Large is compensated by a cooling from Param, with the dominant contribution to Param coming from Meso. A similar leading-order ocean heat balance was found by Gregory (2000), ${ }^{2}$ who also demonstrated the dominant role of the Southern Ocean in maintaining this balance. For the model ensemble mean, SRT (the sum of Large and Meso) tends to make the ocean below roughly $400 \mathrm{~m}$ slightly colder (Fig. 3a). Thus, Small, which must balance SRT at steady state, tends to make it warmer. Two major components contributing to Small are due to small-scale vertical mixing and convection, which, respectively, act to warm and cool the subsurface ocean. Convection takes place at specific locations of the global ocean, while small-scale mixing is typically more evenly distributed in the ocean interior away from rough topography. Since Small is relatively small but positive below about $400 \mathrm{~m}$ (Fig. 3a), we infer that the heating rate associated with small-scale mixing is marginally stronger than the cooling rate associated with convection.

The spreads in the heating rate corresponding to each scale, as given by intermodel standard deviations (STD), increase toward the surface (Fig. 3b). Notably in the 400-1500-m layer, which mostly corresponds to the pycnocline, the spread in Small is considerably lower than the spreads in Large or Meso. Observations and tracer release experiments (e.g., Ledwell et al. 1993) suggest that vertical diffusivity is of the order of $10^{-5} \mathrm{~m}^{2} \mathrm{~s}^{-1}$ over vast ocean regions in the pycnocline, away

\footnotetext{
${ }^{2}$ The AOGCM used by Gregory (2000) did not have eddyinduced advection, so Meso consisted only of isopycnal eddy diffusion.
} 
(a) Rate of heating: 1pctCO2 - piControl

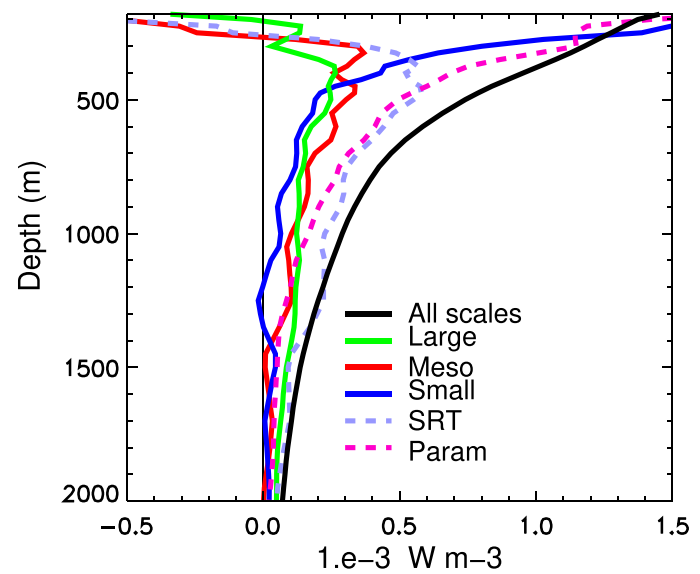

(b) Inter-model STD: 1pctCO2 - piControl

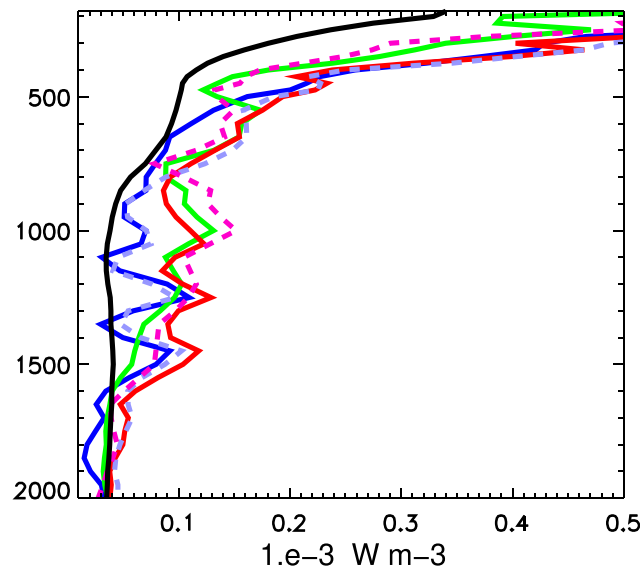

FIG. 4. (a) Global-mean and time-mean (70 years) profiles of changes in heat convergences (1pctCO2 wrt piControl) corresponding to the net heating rate (All scales) and its partitioning into contributions from the resolved circulation (Large), all mesoscale and submesoscale eddy-related processes (Meso), and all diapycnal and other effects (Small). Also shown are the contributions from the SRT (= Large + Meso) and all parameterized (in these AOGCMs) processes (Param = Small + Meso). (b) Intermodel STDs corresponding to the curves in (a).

from regions with rough topography. Such values have now been adopted for background ocean diffusivity in most AOGCMs, which may in part explain the relatively low spread in Small in the 400-1500-m layer. Note that, because of the balances given by Eqs. (7) and (8) and because the spread in the All scales term is negligible in piControl, the spread in SRT is essentially the same as the spread in Small, while the spread in Param is the same as in Large. This implies, in particular, that in the 400-1500-m layer the spread in SRT is as low as it is in Small, adding to the usefulness of the decomposition given by Eq. (7).

Also presented separately in Fig. $3 \mathrm{~b}$ are the spreads corresponding to eddy-induced advection and isopycnal diffusion composing Meso [Eq. (3)]. Their STD profiles closely follow the Meso STD profile, so that their sum is about twice as large as the Meso STD. This result indicates that the uncertainties in heat convergence due to eddy advection and diffusion anticorrelate; that is, models with stronger than average subsurface ocean cooling rate due to eddy-induced heat advection tend to have lower than average subsurface ocean cooling due to eddy isopycnal heat diffusion. This behavior may be expected given the main heat balance in the subsurface ocean (Fig. 3a), in which the ocean interior warming due to Large must be balanced by Meso either through eddy advection or diffusion or both.

The heat balance implied by SRT and Small, with the former cooling the ocean interior below $400 \mathrm{~m}$ and the latter warming it, appears to be consistent with the advective-diffusive balance considered by Munk (1966) and more recently by Munk and Wunsch (1998). A similar result was arrived at by Dias et al. (2020a), who proposed reinterpreting SRT as the advective part of the classical advective-diffusive balance. While a more detailed discussion of this subject is beyond our scope, we note that caution is required when comparing our global SRT and Small profiles to Munk's analysis. In particular, Munk (1966) focused his analysis on the 1-4-km layer in the Pacific Ocean where, as he argued, the warming associated with his inferred layer-mean vertical diffusivity (of the order of $10^{-4} \mathrm{~m}^{2} \mathrm{~s}^{-1}$ ) is consistent with estimates of the bottom water upwelling originating in the Southern Ocean. Munk and Wunsch (1998) arrived at essentially the same conclusion, except reinterpreting Munk's diffusivity estimate as possibly resulting from a small number of concentrated mixing sources. In contrast, much of our global SRT heating profile in Fig. 3a represents a small residual of larger and opposite effects due to wind-driven and eddy-driven processes in the upper $2 \mathrm{~km}$ of the Southern Ocean. The smallness of global-mean SRT implies that the potential energy generated by the large-scale wind-driven circulation, via fluxing more buoyant waters downward (Gnanadesikan et al. 2005; Gregory and Tailleux 2011), is mostly removed by the eddy effects combined in Meso, via fluxing more buoyant waters upward, as also seen in higher-resolution simulations (e.g., Morrison et al. 2013; Griffies et al. 2015).

\section{2) VERTICAL STRUCTURE OF OHU}

In response to $1 \mathrm{pctCO} 2$, the associated heat input to the ocean results in relatively small changes in the vertical heat transport processes (Fig. 4a), with the net effect of these changes leading to OHU. In the balance of Eq. (5), heating of the uppermost ocean is dominated by Small; it becomes less negative above roughly $300 \mathrm{~m}$ and more positive below this depth (Figs. 3a and 4a). Meso controls much of the heating in the 5001000-m layer by becoming less negative. Large also contributes to the subsurface $\mathrm{OHU}$ mostly by becoming more positive. In the Eq. (7) balance, the ocean warming below roughly $400 \mathrm{~m}$ is dominated by SRT, due to both Meso and Large. In the balance given by Eq. (8), the heating above $1000 \mathrm{~m}$ is dominated by the combined effect from all parameterized processes (Param). The spreads across the AOGCMs in the ocean heating rate increase toward the surface (Fig. 4b). Notably, at most depths in the upper ocean there is a higher agreement among the AOGCMs in the net heating rate change (All scales) than in the contributions to it from the individual scales. 
(a) Cumulative from bottom $\mathrm{OHU}$

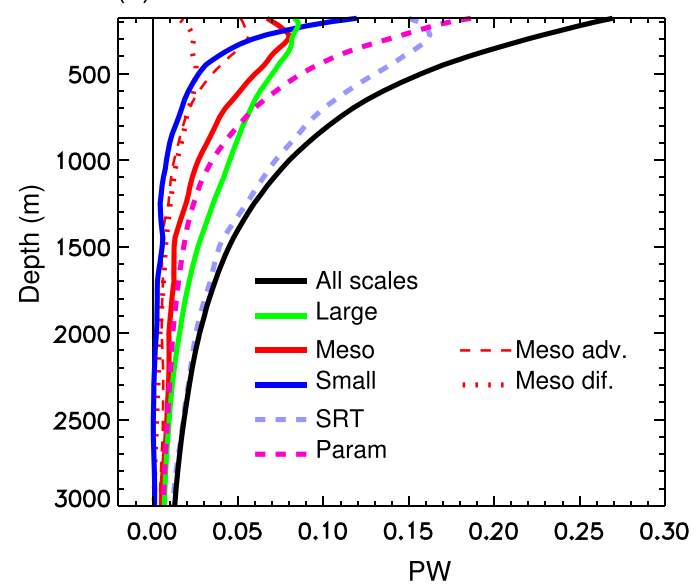

(b) Inter-model STD: OHU

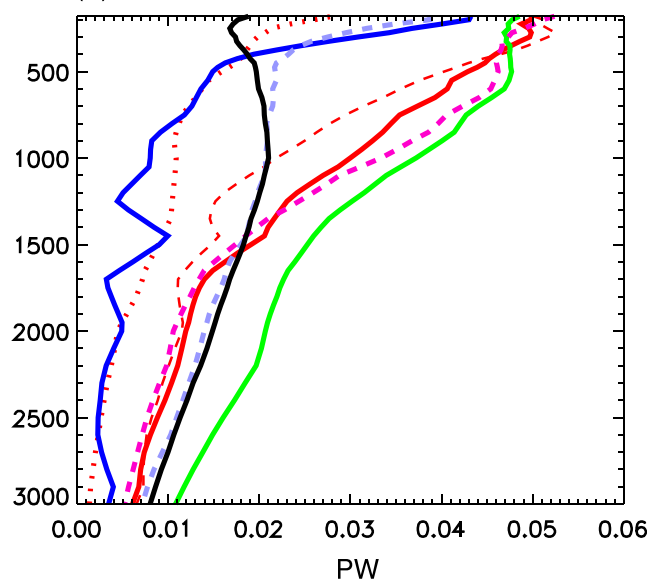

FIG. 5. (a) Integrated horizontally and from the bottom to each depth OHU (i.e., increase in downward heat transport in 1pctCO2 from piControl across each depth) due to all scales (All scales) and its partitioning into contributions from the resolved circulation (Large), all mesoscale and submesoscale eddy-related processes (Meso), and all diapycnal and other effects (Small). Also shown are the contributions from the superresidual transport $($ SRT $=$ Large + Meso $)$ and all parameterized $($ in these AOGCMs $)$ processes $($ Param $=$ Small + Meso $)$, as well as the partitioning of Meso into contributions from eddy advection (Meso adv.) and diffusion (Meso dif.). (b) Intermodel STDs corresponding to the curves in (a).

Another useful view of the global OHU can be obtained by integrating (or accumulating) the heating rates for each layer from the bottom upward (Fig. 5a). This diagnostic quantifies how much heat is taken up by the ocean below a particular depth, and it is equal to the increase in downward heat transport across that depth arising from global warming. We see that all three scales contribute positively to the subsurface OHU. However, below essentially any depth deeper than $400 \mathrm{~m}$ the $\mathrm{OHU}$ is dominated by SRT, with Small being relatively unimportant (Fig. 5a). The contribution of isopycnal diffusion to OHU by SRT is less important than the contribution of the net (resolved plus eddy-induced) advection. However, the contribution of isopycnal diffusion to $\mathrm{OHU}$ by Meso, particularly below about $500 \mathrm{~m}$, is as important as the contribution of eddyinduced advection. Above about $400 \mathrm{~m}$, the contribution from Small is much greater, exceeding the contribution from Meso and Large.

The spreads corresponding to the cumulative OHU profiles in Fig. 5a are quantified in Fig. 5b. The STD corresponding to the All scales profile is rather uniform with depth. This feature indicates that the OHU below any depth is roughly equally uncertain. From the variance (var) profiles corresponding to the individual scales it follows that, in particular,

$$
\begin{gathered}
\operatorname{var}(\text { SRT })<\operatorname{var}(\text { Large })+\operatorname{var}(\text { Param }), \quad \text { and } \\
\operatorname{var}(\text { SRT })<\operatorname{var}(\text { Large })+\operatorname{var}(\text { Meso }),
\end{gathered}
$$

indicating an anticorrelated (compensating) behavior between Large and Param and between Large and Meso [since Small and its spread are small below $400 \mathrm{~m}$, the near-global compensation implied by Eq. (15a) is principally between Large and Meso, as implied by Eq. (15b)]. It is also notable that the spread in the OHU by isopycnal diffusion is smaller than the spread in the OHU by eddy-induced advection.

The spread in the near-global OHU, as given by the uppermost STD values corresponding to All scales (Fig. 5b), is relatively small; that is, it is smaller than the STDs of the individual scales contributing to the global OHU. The finding that the model spread in global OHU is relatively small is consistent with the analysis based on a larger ensemble of CMIP5 and CMIP6 AOGCMs presented in J. M. Gregory et al. (2020, unpublished manuscript). In other words, the models are more similar in their simulated net OHU than in the processes through which the heat anomalies are transported into the oceanic interior. This result implies that global OHU tends to self-adjust to the uncertainties in the representation of unresolved ocean physics in AOGCMs.

To put the finding that global OHU varies little across the models into context, Fig. 6a compares the model-mean depth profiles and spreads of two quantities, the first being the OHU below $z, \mathrm{OHU}(z)$, corresponding to each model $i=1, \ldots, N$ $(=11)$ and normalized by the model-mean effective temperature change in the layer above $z,\langle\Delta T\rangle(z)=N^{-1} \sum_{i} \Delta T_{i}(z)$,

$$
\mathscr{E}_{1}^{i}(z)=\frac{\mathrm{OHU}_{i}(z)}{\langle\Delta T\rangle(z)},
$$

and the other is $\mathrm{OHU}_{i}(z)$ normalized instead by the model's own temperature change in the layer above $z$

$$
\mathscr{E}_{2}^{i}(z)=\frac{\mathrm{OHU}_{i}(z)}{\Delta T_{i}(z)},
$$

where $\Delta T(z)$ is given by the heat content change in the layer above $z$, divided by the layer's thickness, volumetric heat capacity of seawater, and ocean surface area. We consider 

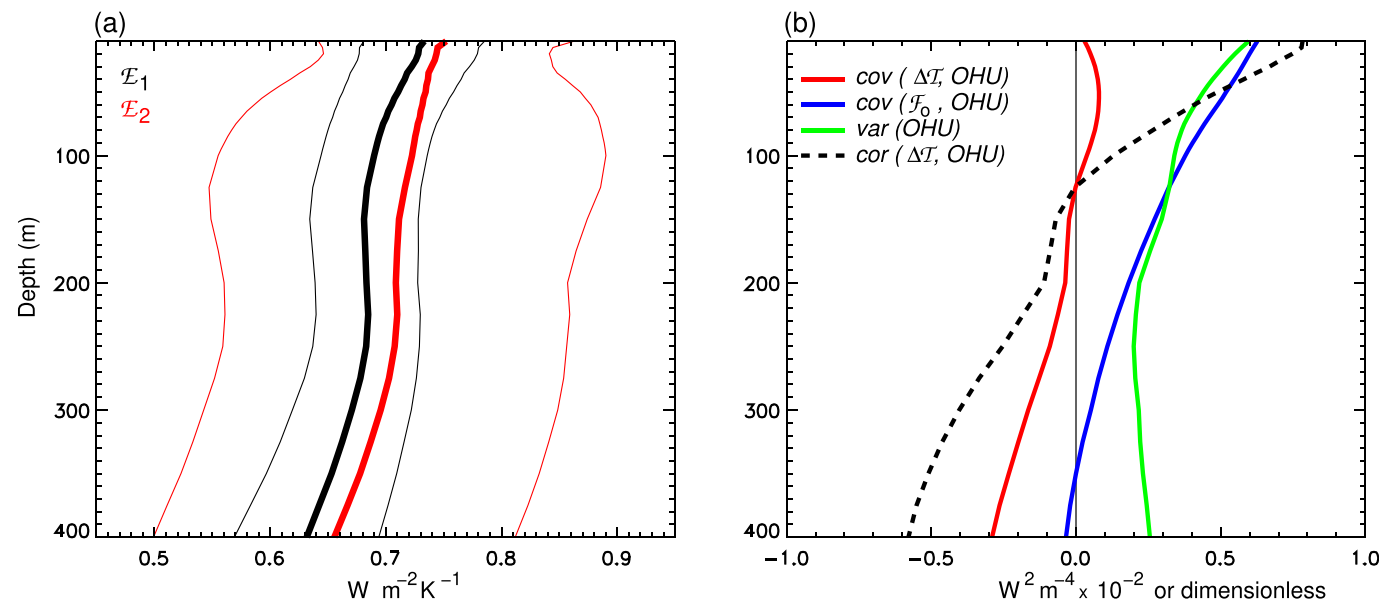

FIG. 6. (a) Depth profiles of $\mathscr{E}_{1}$ and $\mathscr{E}_{2}$ in the upper ocean, given by Eqs. (16) and (17), with thick lines corresponding to model-mean quantities and thin lines corresponding to model-mean \pm 1 intermodel STD. (b) Depth profile of covariance between the heat convergence change in the upper ocean above a particular depth, $\Delta \mathscr{T}(z)$, and $\mathrm{OHU}$ below this depth, $\mathrm{OHU}(z)[\operatorname{cov}(\Delta \mathscr{T}, \mathrm{OHU})]$ and its two components: covariance between the surface heat flux anomaly $\mathscr{F}_{0}$, and $\mathrm{OHU}(z)$ and variance of $\mathrm{OHU}(z)$ (see appendix B). Dashed line shows correlation between $\Delta \mathscr{T}(z)$ and $\mathrm{OHU}(z)$.

$\mathscr{E}_{2}(z=-10 \mathrm{~m})$ as a proxy for OHU efficiency (OHUE)—one of the more important characteristics of climate response to $\mathrm{CO}_{2}$ increase in AOGCMs (e.g., Kuhlbrodt and Gregory 2012), more traditionally defined as the ratio of the net heat flux into the climate system to the global surface air temperature change. Kuhlbrodt and Gregory (2012) found that OHUE varies considerably, by a factor of 2 across the AOGCMs they analyzed. This behavior is consistent with our calculation of $\mathscr{E}_{2}(z=-10 \mathrm{~m})$, which ranges from 0.61 to $0.96 \mathrm{~W} \mathrm{~m}^{-2} \mathrm{~K}^{-1}$, with the model ensemble mean of $0.75 \mathrm{~W} \mathrm{~m}^{-2} \mathrm{~K}^{-1}$ and standard deviation of $0.11 \mathrm{~W} \mathrm{~m}^{-2} \mathrm{~K}^{-1}$. Thus, the coefficient of $\mathscr{E}_{2}$ variation (ratio of model ensemble standard deviation to ensemble mean) is $15 \%$, increasing to $22 \%$ for $\mathscr{E}_{2}(z=-100 \mathrm{~m})$. For comparison, the ensemble standard deviation of $\mathscr{E}_{1}(z=-10 \mathrm{~m})$ is only $0.05 \mathrm{~W} \mathrm{~m}^{-2} \mathrm{~K}^{-1}$ and the coefficient of its variation is $7 \%$. Therefore, we conclude that most of the intermodel variation in $\mathscr{E}_{2}(z)$ arises from uncertainty in the ocean temperature change above $z$ rather than in OHU below $z$. A similar conclusion regarding OHUE variation can be drawn from the analysis presented in Kuhlbrodt and Gregory (2012).

Moreover, the correlation between the change in heat convergence in an upper-ocean layer of thickness $z$, which drives the global-mean temperature change $\Delta T(z)$ of the layer, and the $\mathrm{OHU}(z)$ below it decreases with depth and becomes negative at about 130-m depth (Fig. 6b). This anticorrelated behavior between $\Delta T(z)$ and $\mathrm{OHU}(z)$, for a thick enough upper layer, arises because the covariance between the surface heat flux anomaly and $\mathrm{OHU}(z)$ decreases with depth, while the variance of $\mathrm{OHU}(z)$ is more uniform (Fig. 6b; see appendix B). Thus, a stronger warming of the upper ocean, such as in response to $\mathrm{CO}_{2}$ increase, does not necessarily imply a stronger warming of the ocean below it. This behavior is unlike that in some two-layer box models of OHU, in which heat content change in the lower layer ("deep ocean") is commonly assumed to be proportional to temperature change in the upper layer. Instead, Fig. 6b suggests that for a thick enough upper layer $(100-200 \mathrm{~m})$ its temperature change is not strongly related to the net temperature change in the ocean below it and may even anticorrelate with it. In fact, despite the strong correlation between $\mathrm{OHU}$ and temperature change in the upper $\sim 50 \mathrm{~m}$ of the ocean (Fig. $6 \mathrm{~b}$; dashed line), OHU is not proportional to the near-surface temperature change, with much of the former being independent of the latter (not shown). A more detailed analysis of the relationships between OHUE, OHU, surface temperature change and the strength of the Atlantic meridional overturning circulation (AMOC) is presented in J. M. Gregory et al. (2020, unpublished manuscript).

Moreover, the diffusive nature of heat transfer from the surface to subsurface ocean, which is also commonly assumed in box models of OHU, is not supported by the AOGCMbased heat budget analysis in density space presented in section $3 b$.

\section{3) Regional structure of OHU}

One conclusion from our analysis so far is that ocean physics and dynamics operating at all scales contributes to subsurface $\mathrm{OHU}$, while the dominance of a particular scale or scales depends on depth. This result raises some further questions. In particular, what are the contributions from different regions to the global subsurface OHU due to Large, Meso, and Small? Where in the ocean do the largest contributions to Param come from and how are they partitioned between Meso and Small? How is the global value of Large set and what are the contributions to it from different oceans? What are the regions of largest OHU uncertainties?

Some answers can be obtained from Fig. 1, which presents spatial structure of $\mathrm{OHC}$ change corresponding to the heat budget decomposition given by Eq. (7). In particular, the 
subsurface ocean warming due to Small results from changes in mid- and high-latitude regions (Fig. 1b). The localization of these changes to (mostly) the northern North Atlantic and Southern Ocean suggests their convective origin; that is, a weakening of convective mixing in response to surface buoyancy input and increased stratification, such as in 1 pctCO2, tends to make the local subsurface ocean warmer. This interpretation of Fig. 1b is consistent with Exarchou et al. (2015) and Kuhlbrodt et al. (2015), who considered a more detailed separation of Small into several contributors. In contrast, the changes in SRT lead to cooling in the subpolar Atlantic and warming in, for example, the low-latitude Atlantic (Fig. 1c). As we shall see, this north-south heat redistribution in the Atlantic is related to the weakening of the AMOC (Fig. 8a), which causes less heat convergence in the subpolar North Atlantic and less heat divergence in the Atlantic at lower latitudes.

In addition to the basin-scale $\mathrm{OHC}$ changes, SRT also causes some important local OHC changes, such as an enhanced warming in the Gulf Stream region and its extension. Integrated below $200 \mathrm{~m}$, the heat input of $4.6 \mathrm{TW}(1 \mathrm{TW}=$ $10^{12} \mathrm{~W}$ ) to the region (green box in Fig. 1c) is dominated by advective component of SRT. Given the narrowness of the Gulf Stream region, its warming due to SRT is perhaps reinforced by a slight northward shift in the mean position of the current that could be associated with the weakening of AMOC (Saba et al. 2016). A more in-depth analysis needs to be performed to confirm this suggestion, preferably based on higherresolution models. Overall, these results suggest that, while much of the OHC change in the North Atlantic can be explained by the heat taken up as a passive tracer (Gregory et al. 2016; Couldrey et al. 2021), changes in ocean dynamics and the associated heat redistribution also play an important role.

The regions of largest uncertainties in the spatial structure of OHC change are the subpolar North Atlantic, Arctic Ocean, and Southern Ocean (Fig. 1d). These are also the regions of largest uncertainties in dynamic sea level changes (e.g., Gregory et al. 2016; Couldrey et al. 2021). Therefore, while the global OHU is rather similar across the models [section $3 \mathrm{a}(2)$; J. M. Gregory et al. 2020, unpublished manuscript], reducing the uncertainties in the regional $\mathrm{OHC}$ changes (and, hence, in spatial sea level changes) would require a more accurate representation of ocean dynamics and unresolved physics than in the analyzed AOGCMs.

To obtain further insight, Figs. 7a and $7 \mathrm{~b}$ present OHU accumulated from the south (OHC change) and its contributions from the considered scales, for the global ocean (Fig. 7a) and separately for the Atlantic Ocean (Fig. 7b). The net global OHU below $200 \mathrm{~m}$ is dominated by parameterized processes, as can be deduced from the northernmost values in Fig. 7a (see also the uppermost values in Fig. 5a). This feature is consistent with Exarchou et al. (2015). A major contribution to global Param comes from its changes north of $40^{\circ} \mathrm{N}$, particularly in the Atlantic (Fig. 7b). This region is where the subsurface ocean warming due to the parameterized processes accounts for roughly half of their contribution to the global OHU (Figs. 7a,b; dashed magenta), but this warming is nearly fully compensated by cooling due to changes in the large-scale heat advection (Figs. 7a,b; green). As a result, the All scales line flattens north of $40^{\circ} \mathrm{N}$. This near compensation between contributions from Param and Large to the $\mathrm{OHC}$ change in the North Atlantic appears to be related to two main processes (Fig. 8): 1) weakening of the AMOC and the associated northward heat transport which, as part of Large, tends to decrease the heat content north of $40^{\circ} \mathrm{N}$; and 2) weakening of deep convection that, as part of Param, tends to increase the subsurface heat content locally through the increase of heat sequestered at depth. Thus, if the ocean north of $40^{\circ} \mathrm{N}$ in the Atlantic were excluded from the analysis, then Large would become almost as important as Param in the budget given by Eq. (8), while SRT would contribute twice as much as Small to the All scales OHU in Eq. (7).

We also note that if the whole water column were considered, rather than only the ocean below 200-m depth, then the $\mathrm{OHU}$ associated with processes that transport heat only vertically (e.g., convection and vertical diffusion) would integrate to zero. In that case, much of the subpolar North Atlantic cooling associated with the weakening of horizontal heat convergence in the region would instead be balanced by enhanced heat input (or less heat loss) at the surface. Thus, since Small is the most important term in OHU near the surface (Figs. 4a and 5a), it is the principal means by which the change in surface heat flux is transmitted to the ocean below $200 \mathrm{~m}$. Indeed, Fig. 1b resembles the surface heat flux change in 1 pctCO2 with respect to piControl (e.g., see Fig. $2 b$ in Gregory et al. 2016).

The changes in Large not only make the Atlantic north of about $40^{\circ} \mathrm{N}$ colder, but also make the rest of it warmer (Fig. 7b). However, while Large plays an important role in this northsouth heat redistribution within the Atlantic, its contribution to the net Atlantic heat content change north of $30^{\circ} \mathrm{S}$ is relatively small on the model mean (this property can be deduced from the corresponding northernmost value in Fig. 7b; green line). Thus, an interesting result is that, while the whole Atlantic Ocean accounts for about $30 \%$ of the net subsurface OHU, this OHU is mostly due to Param rather than Large (Fig. 7b). Weak stratification in the northern North Atlantic and the associated deep convective mixing intimately link Param and Large to form the basin-scale AMOC, which takes up heat via Param and redistributes it southward via Large. This finding is supported by the heat budget analysis presented in Dias et al. (2020b).

Meso, which is part of Param, importantly contributes to the North Atlantic OHU, particularly between $40^{\circ}$ and $60^{\circ} \mathrm{N}$ (Fig. 7b). When combined with Small, it more than offsets the negative contribution from Large to $\mathrm{OHC}$ change in the region. Exarchou et al. (2015) also found a contribution from eddy processes to heat uptake in the North Atlantic and attributed it to changes in isopycnal temperature gradients and shallower isopycnal slopes in their models. In the models we analyze, the increased subsurface heat convergence due to Meso in the North Atlantic of about $0.025 \mathrm{PW}\left(1 \mathrm{PW}=10^{15} \mathrm{~W}\right)$ is dominated by changes in eddy-induced heat advection. The contribution of isopycnal diffusion to North Atlantic OHU is less important (Fig. 7b), subject to uncertainties (Fig. 7d). It should also be noted that in the Labrador Sea, eddy heat 
(a) Cumulative from south: Global

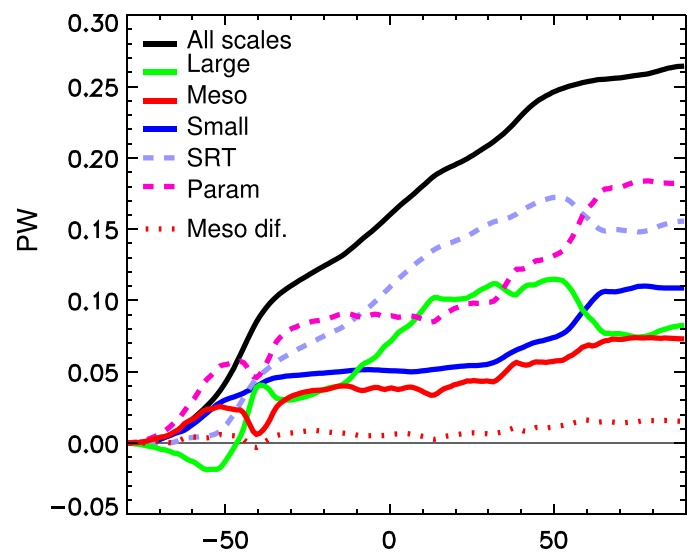

(c) STDs corresponding to panel (a)

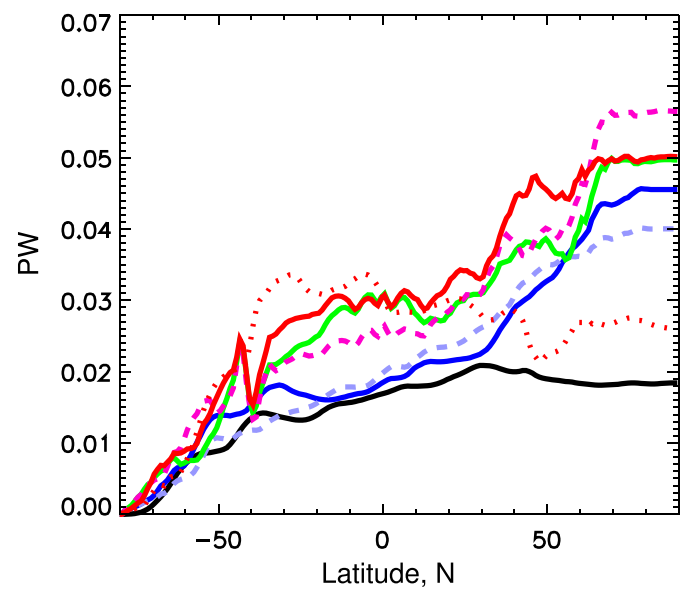

(b) Cumulative from 30S: Atlantic

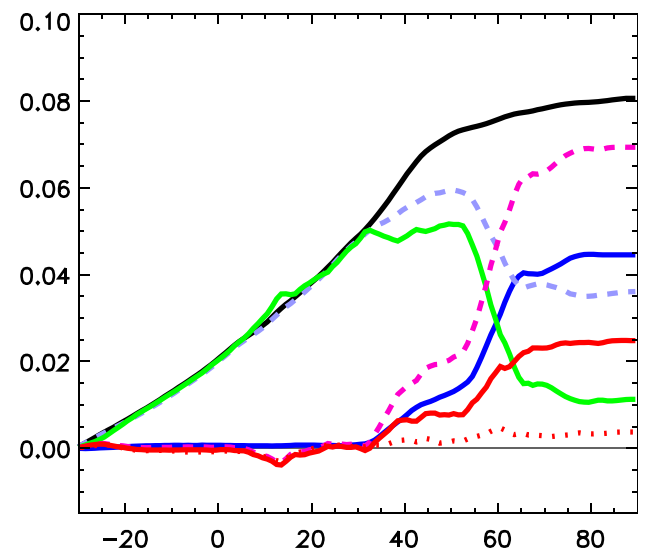

(d) STDs corresponding to panel (b)

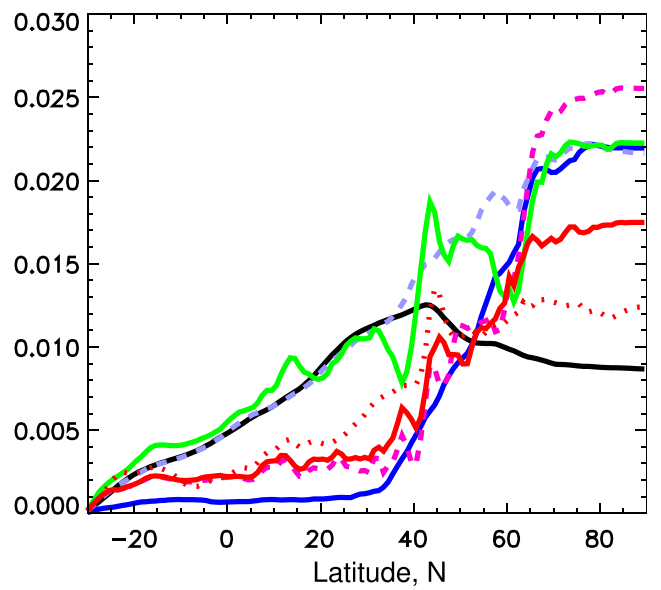

FIG. 7. (a) Integrated zonally and vertically below 200-m depth and from the south to each latitude (i.e., cumulative from the south) OHU (PW) due to all scales (All scales) and its partitioning into contributions from the resolved circulation (Large), all mesoscale and submesoscale eddy-related processes (Meso) and all diapycnal and other effects (Small). Also shown are the contributions from the superresidual transport (SRT $=$ Large + Meso), all parameterized (in these AOGCMs) processes (Param = Small + Meso), and diffusive component of Meso (Meso dif.). (b) As in (a), but for the Atlantic Ocean only and north of $30^{\circ} \mathrm{S}$. (c),(d) Intermodel STDs corresponding to the curves in (a) and (b), respectively.

convergence associated with lateral fluxes of warmer water from the boundary currents into the interior is thought to be the principal means balancing the local heat loss to the atmosphere (e.g., Khatiwala and Visbeck 2000). In addition, eddies typically flux heat upward, cooling the subsurface Fig. 3a. Taking, for example, the estimate of Khatiwala and Visbeck (2000) for the local eddy-induced overturning in the Labrador Sea of $1.3 \mathrm{~Sv}\left(1 \mathrm{~Sv} \equiv 10^{6} \mathrm{~m}^{3} \mathrm{~s}^{-1}\right)$ and assuming, also following them, that it operates on the horizontal temperature contrast of $2 \mathrm{~K}$ gives about $0.01 \mathrm{PW}$ for the associated upward eddy heat transport. Thus, the subsurface warming by Meso in the North Atlantic could be explained, at least in part, by a decrease in this eddy-induced transport, as may be expected in response to the increased stratification (decreased isopycnal slopes) and decreased mixed layer depth in 1pctCO2 (Fig. 8c).
The low-latitude $\mathrm{OHC}$ change, between $30^{\circ} \mathrm{S}$ and $30^{\circ} \mathrm{N}$, accounts for about $35 \%$ of the net subsurface OHU, with Large making the largest contribution (Fig. 7a). Using an OGCM forced with the Flux-Anomaly-Forced Model Intercomparison Project (FAFMIP) surface perturbations corresponding to $2 \times$ $\mathrm{CO}_{2}$ (see Gregory et al. 2016), Dias et al. (2020b) estimate that $65 \%$ of the OHC change at low latitudes is due to the redistribution of heat associated with SRT, dominated by the largescale advection. The contributions from Meso and Small are relatively weak (i.e., the red and blue lines in Figs. $7 \mathrm{a}$ and $7 \mathrm{~b}$ are essentially flat at the low latitudes). This feature is unlike in one-dimensional upwelling-diffusion models, in which diapycnal diffusion is the main process of OHU (e.g., Raper et al. 2001). Moreover, the global low-latitude OHU due to Large is dominated by its changes in the Atlantic Ocean (Figs. 7a,b), with the associated advective convergence being latitudinal 

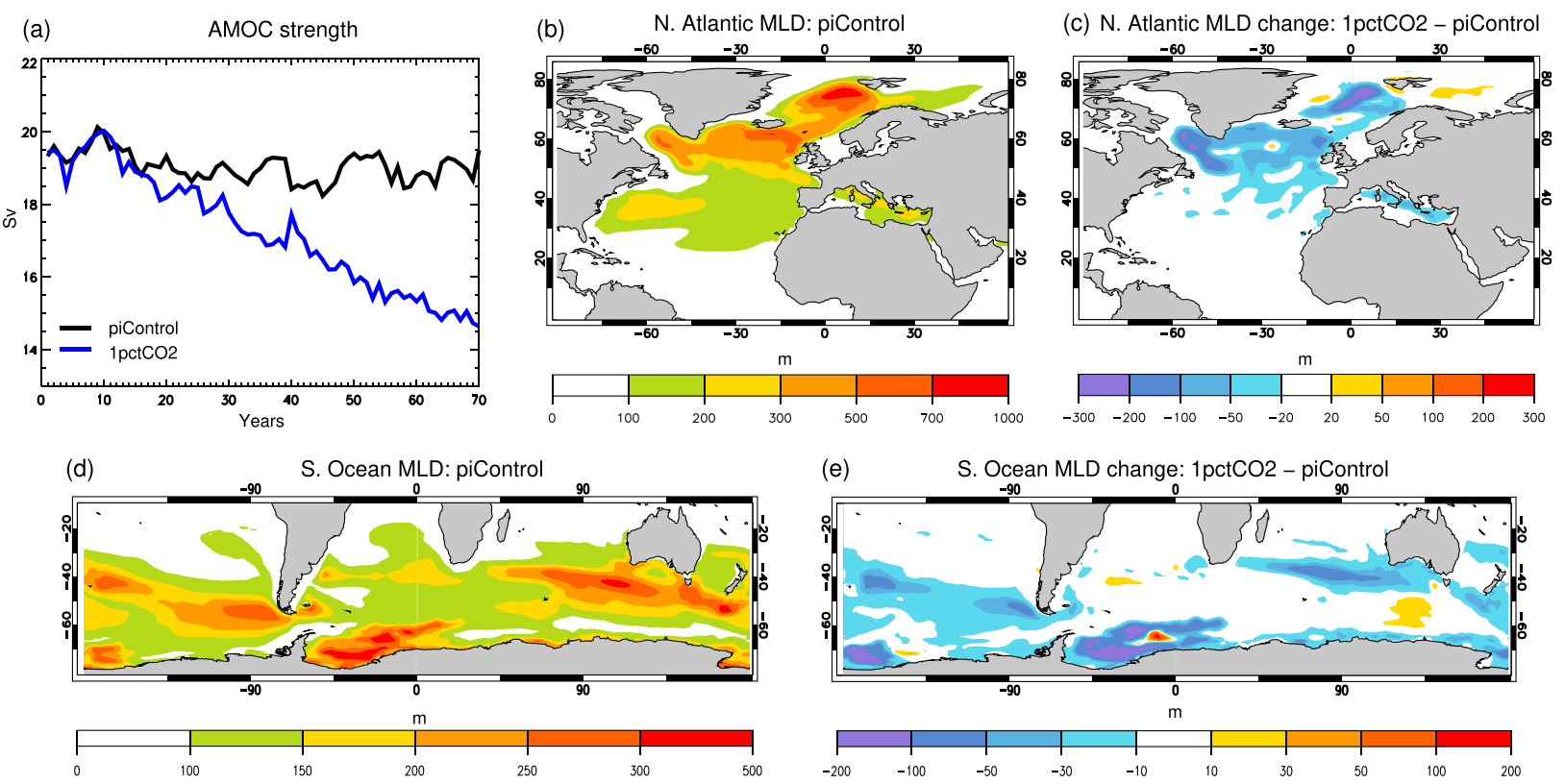

FIG. 8. Model-mean (a) time series of the AMOC maximum in piControl and 1pctCO2, (b) winter (January-March) mixed layer depth (MLD) in the North Atlantic in piControl, (c) the North Atlantic MLD change in 1pctCO2 with respect to piControl, (d) summer (JulySeptember) MLD in the Southern Ocean in piControl, and (e) the Southern Ocean MLD change in 1pctCO2 with respect to piControl. The MLD changes in (c) and (e) represent averages for years 61-70 of 1pctCO2. The MLD corresponds to the mlotst variable (see Griffies et al. (2016) for details). For two models, HadCM3 and HadGEM2-ES, mlotst was estimated using monthly temperature and salinity.

redistribution of heat, rather than low-latitude heat uptake (as assumed by the upwelling-diffusion model).

The ocean south of $30^{\circ} \mathrm{S}$ accounts for about $40 \%$ of the net subsurface $\mathrm{OHU}$ in the model mean (Fig. 7a). In this region, the contribution to OHU from the different scales strongly depends on latitude. Perhaps a preferable frame for analyzing an integrated heat uptake in the Southern Ocean would be along streamlines of depth-integrated transport. Nevertheless, we can conclude that Meso and Small (and, hence, Param) are the largest contributors to the (positive) OHU south of $50^{\circ} \mathrm{S}$. Large opposes Meso and Small south of $50^{\circ} \mathrm{S}$, but contributes considerably to the (positive) $\mathrm{OHU}$ between $50^{\circ}$ and $40^{\circ} \mathrm{S}$. The latitudinal structure of the Large contribution to the Southern Ocean OHU is broadly consistent with the structure of winddriven upwelling and downwelling in the region.

The spatial pattern of OHC change in the Southern Ocean is nonuniform, with stronger warming in the Atlantic Ocean and Indian Ocean sectors than in the Pacific Ocean sector (Fig. 1a), consistent with Gregory et al. (2016, their Fig. 9d). This pattern is related to the positive contribution from SRT in the Atlantic and Indian sectors (Fig. 1c). In the Atlantic sector, the local $\mathrm{OHU}$ is likely reinforced by advective heat redistribution to the south (Fig. 7b) associated with the weakening of the AMOC (Fig. 8a). In the Indian sector, decomposition of the net $\mathrm{OHC}$ change into contributions from the added and redistributed heat shows enhanced contribution from the latter south of about $40^{\circ} \mathrm{S}$ (Gregory et al. 2016; Couldrey et al. 2021); some of it might be connected with the Atlantic Ocean (Dias et al. 2020b). In the Pacific sector the Atlantic warming signal becomes weaker, and so does the contribution of SRT to the local OHC change (Figs. 1a,c). In the southeast Pacific, upstream of the Drake Passage, the local warming is dominated by the changes in Small (Fig. 1b). This region has been identified as a key site of Antarctic Intermediate Water (AAIW) and Subantarctic Mode Water (SAMW) formation characterized by deep mixed layers, with another site of SAMW formation located in the southern Indian Ocean (see Naveira Garabato et al. 2009, and references therein). The AOGCMs do simulate deep mixed layers in these regions of the Southern Ocean in piControl (Fig. 8d), with the mixing becoming less deep in 1pctCO2 (Fig. 8e). The latter indicates a weakening of convective mixing, induced by stronger stratification, leading to the local subsurface warming due to Small (Fig. 1b). It can therefore be concluded that the net OHC change in the southeast Pacific results from a subtle interplay between the contributions from Small, making it warmer, and from SRT tending to make it colder. The latter could be related to an enhanced upwelling and northward flux of relatively cold water south of $60^{\circ} \mathrm{S}$ in response to $\mathrm{CO}_{2}$ (e.g., Saenko et al. 2005).

The spreads corresponding to the OHC changes accumulated from the south in Figs. $7 \mathrm{a}$ and $7 \mathrm{~b}$ are quantified in Figs. 7c and $7 \mathrm{~d}$. The spread in the (near) global OHU, as given by the northernmost STD value corresponding to All scales in Fig. 7c, is smaller than the STDs of Large, Meso, and Small (cf. Fig. 5b). North of about $40^{\circ} \mathrm{N}$ the spreads in the individual scales increase, mostly due to their increase in the Atlantic (Fig. 7d), while the STD corresponding to All scales remains relatively uniform. There is a better agreement among the AOGCMs in the net OHU, globally and in the Atlantic, than in the individual scales/processes. Again, this behavior implies a 
TABLE 3. Contribution of physics and dynamics at different scales to ocean heat uptake (PW; $1 \mathrm{PW}=10^{15} \mathrm{~W}$ ) below several indicated depths and below the thermocline depth (TD). The numbers represent model-mean values for years $61-70$ of $1 \mathrm{pctCO} 2$ with respect to piControl and correspond to the models for which the heat tendency diagnostics were available as monthly averages (see Table 1).

\begin{tabular}{lcccc}
\hline \hline & $200 \mathrm{~m}$ & $400 \mathrm{~m}$ & $700 \mathrm{~m}$ & TD \\
\hline Large & 0.13 & 0.17 & 0.15 & 0.26 \\
Meso & 0.11 & 0.12 & 0.06 & 0.01 \\
Small & 0.20 & 0.07 & 0.04 & 0.07 \\
All scales & 0.44 & 0.36 & 0.25 & 0.34 \\
\hline
\end{tabular}

degree of compensation between different scales in their contribution to $\mathrm{OHU}$.

\section{4) OHU BELOW THE THERMOCLINE}

So far, we have discussed the OHU below fixed depth levels. In the next section the focus is on the OHU projected onto potential density surfaces. Here, as an intermediate step, we briefly discuss the OHU below the seasonal thermocline. Different criteria are used to define the depth of seasonal thermocline, such as based on vertical temperature gradient or on the depth of specific isotherms (e.g., $20^{\circ} \mathrm{C}$ ). The former requires a high enough vertical resolution and may not be suitable for all models, while the latter is not applicable everywhere in the ocean (the corresponding heat budget represents a special case of the OHU in temperature or density coordinates). Here we employ a simple criterion that avoids these difficulties and, at the same time, helps to identify the major processes fluxing the $\mathrm{CO}_{2}$-induced heat anomalies from the upper ocean and high-latitude regions to the low-latitude oceanic interior. The criterion is based on the depth where the potential temperature differs from the temperature at the surface by more than $0.5^{\circ} \mathrm{C}$, which is representative of the seasonal thermocline depth (Tomczak and Godfrey 1994). As defined this way, the thermocline depth is typically within $100-300 \mathrm{~m}$ between $35^{\circ} \mathrm{S}$ and $35^{\circ} \mathrm{N}$, but is much deeper at middle and high latitudes, as intended.

The key findings are summarized and compared with OHU below several fixed depths in Table 3. In particular, the net OHU below the seasonal thermocline (All scales) is similar to that below 400-m depth. It is dominated by Large, representing the propagation of heat anomalies from both the upper ocean and high-latitude oceans toward the low-latitude regions. Small also plays a role and is the same as OHU due to Small below 400-m depth (although this does not necessarily imply the same physics). The main difference between the processes driving the OHU below 400-m depth and below the thermocline is that in the latter case the contribution from Meso is quite small. One reason for this behavior, as already noted, is that the thermocline (as defined the way described above) penetrates to large depths at middle and high latitudes, including in most of the Southern Ocean. This deep thermocline effectively excludes the Southern Ocean eddy effects from a direct contribution to OHU below the thermocline. However, the combined contribution of Large and Meso (i.e., SRT) to OHU below the thermocline is similar to that below fixed depth levels in the upper ocean.

\section{b. Potential density space $\mathrm{OHU}$ analysis}

A heat budget in potential density space (density referenced to $0 \mathrm{dbar}$ ), following the procedure described in section $2 \mathrm{c}$ (see also appendix A), provides further insight on the OHU process. In piControl (Fig. 9a), surface heat loss at densities larger than about $25 \sigma_{\theta}$ (e.g., regions of western boundary currents and, at higher densities, deep water formation regions) is resupplied by diapycnal mixing processes included in Small and by the resolved advection in Large, particularly at the highest density classes. The heating by Large is partly offset by Meso due to the eddy-induced advection of heat (isopycnal diffusion of temperature, which is also included in Meso, cannot flux temperature across isopycnals). The time-mean net ["All scales" or $\mathscr{H}(\rho)$, as given by Eq. (11)] is relatively small in piControl since it is close to a statistical steady state in piControl. However, it is not negligible. For example, the associated warming of waters denser than $26.5 \sigma_{\theta}$ is of the order of $0.1 \mathrm{PW}$.

Taking the difference between $1 \mathrm{pctCO} 2$ and piControl gives the net $\mathrm{OHU}\left[=\mathscr{H}\left(\rho_{\text {lightest-water }}\right)\right]$ of about $0.65 \mathrm{PW}$ and shows the contributing processes (Fig. 9b). For densities lower than $25.5 \sigma_{\theta}$ the shape of the individual curves reflects, in part, the creation of new light density classes in response to $\mathrm{CO}_{2}$ increase and the associated warming. It should be noted, however, that waters with densities less than $25.5 \sigma_{\theta}$ are mostly confined to $35^{\circ} \mathrm{S}-35^{\circ} \mathrm{N}$ and, on average, do not penetrate deeper than $200 \mathrm{~m}$.

Most of the heat uptake takes place at densities larger than $25.5 \sigma_{\theta}$. This behavior is expected since waters with these densities occupy most of the ocean volume. Furthermore, for $\sigma_{\theta}>25.5$, the net OHU line closely follows the heat accumulation given by the surface heat flux anomaly (solid and dashed lines in Fig. 9b), with the contributions from different scales being relatively small and nearly cancelling each other. This result suggests that most of the OHU can be characterized as an isopycnal process. This behavior is unlike one-dimensional upwelling-diffusion models, in which diapycnal (i.e., vertical) diffusion is the main process for OHU (e.g., Raper et al. 2001). Instead, in the analyzed AOGCMs, most OHU occurs through the SRT (= Large + Meso). This result follows since Small contains only diapycnal processes, while SRT is represented by both diapycnal and isopycnal processes. However, since diapycnal processes do not contribute much to the OHU at densities larger than $25.5 \sigma_{\theta}$, we infer that isopycnal transport processes as part of the SRT perform the bulk of the heat uptake, and they do so by linking the interior $\mathscr{H}(\rho)$ to heat input at the surface [Eq. (10)].

Moreover, applying the diathermal framework (i.e., replacing in Eq. (10) potential density with temperature) leads to a similar result. Namely, for temperatures colder than $25^{\circ} \mathrm{C}$, the net OHU ("All scales" anomaly) closely follows the surface heat flux anomaly (not shown), implying that most of the OHU is isothermal. This result, combined with the analysis in 
(a)

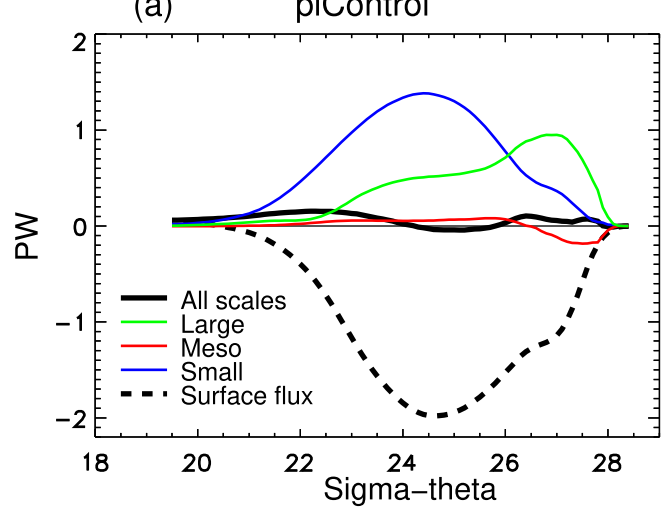

(c) Density framework

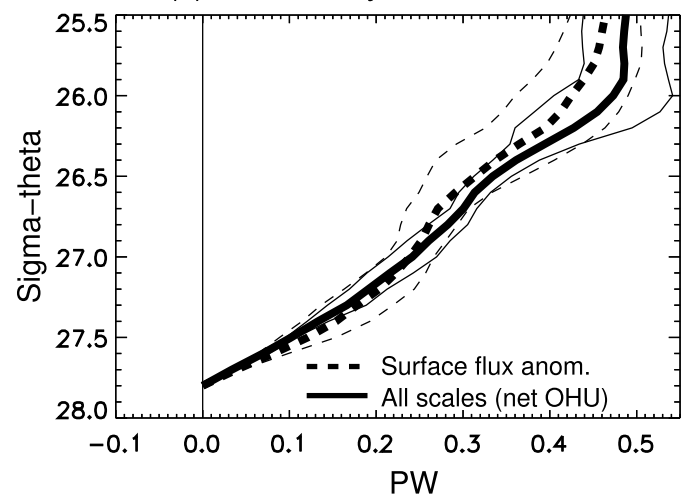

(b) $\quad 1$ pctCO2 - piControl

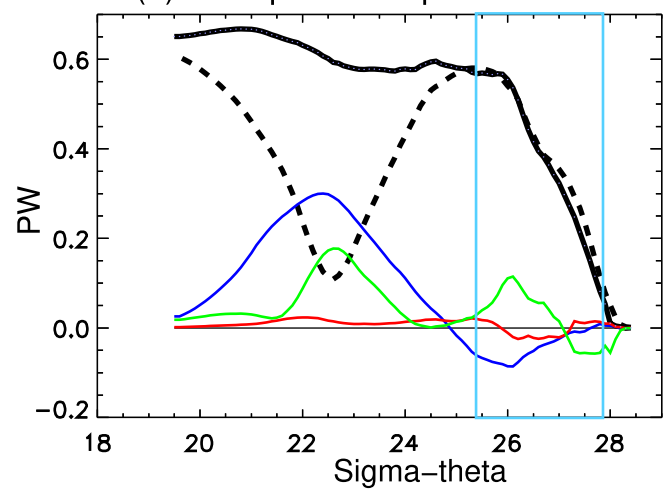

(d) Depth framework

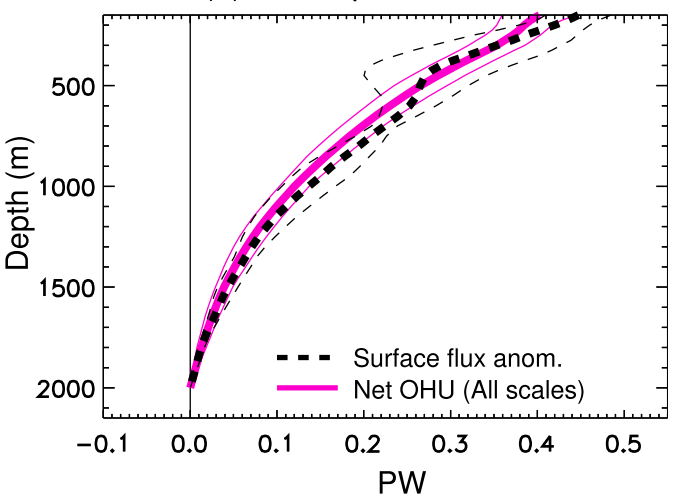

FIG. 9. Model-mean heat budget in potential density $\sigma_{\theta}$ (referenced to the surface) coordinates (see text for details) corresponding (a) piControl (positive values correspond to heat convergence within higher density classes), (b) its change (1pctCO2 wrt piControl), and (c) plotted separately the surface heat flux anomaly and net OHU ("All scales") corresponding to the (rotated) light-blue box in (b), plotted relative to their values at $\sigma_{\theta}=27.8$. (d) Projection of the surface flux anomaly from density space in (c) onto mean depths of the corresponding isopycnals, along with the mean profile of net $\mathrm{OHU}$ computed using 3D temperature tendencies directly from the ocean interior; both quantities are plotted relative to their values at 2000-m depth, which roughly corresponds to the model-mean depth of the $\sigma_{\theta}=27.8$ surface. The plots correspond to the model-mean (see Table 1 ) and time-mean quantities for years 61-70 of 1pctCO2 and the corresponding years of piControl. In (c) and (d), thick lines represent model-mean quantities, while thin lines represent the corresponding \pm 1 intermodel STDs.

section 3a(2) (Fig. 5a; see also Fig. 7a), further suggests that it is the advective component of SRT that accounts for most of the OHU. We make this inference since there is no heat diffusion along isothermal surfaces.

It also follows from Fig. 9b that, if the flows in the ocean interior were mostly along isopycnals, such as expected away from regions of strong diapycnal mixing, then it should be possible to reconstruct the vertical structure of the OHU profile from the surface heat flux anomaly [i.e., using the last term in Eq. (10)]. We demonstrate this reconstruction by projecting the surface heat flux anomaly from density space for 25.5-27.8 $\sigma_{\theta}$ (Fig. 9c) onto the mean depths of the corresponding density surfaces for the 150-2000-m layer (Fig. 9d); that is, where most of the OHU takes place and where $\sigma_{\theta}$ is mostly monotonic with depth. Thus, because of the near isopycnal nature of the OHU process, the input of heat at the surface for $\sigma_{\theta}>25.5$ and its penetration into the ocean interior within the same density classes is reflected in the global profile of $\mathrm{OHU}(z)$ (Fig. 9d). This process is schematically illustrated in Fig. 10.

It should be noted that one way to reconcile the isopycnal and horizontal averaging approaches of $\mathrm{OHU}$ analysis is to constrain the integration in Eq. (10) to the ocean volume below some depths. In this case the OHU below, for example, $100 \mathrm{~m}$ depth is dominated by Small, while the OHU below 400-m depth is dominated by SRT (not shown), as expected based on the results in section $3 \mathrm{a}(1)$.

\section{Discussion and conclusions}

Using heat budget diagnostics from a set of coarseresolution (nonmesoscale eddying) AOGCMs run in preindustrial control (piControl) and an idealized (1pctCO2) climate change experiment, we study the contribution to OHU arising from parameterized ocean physical processes and resolved dynamical features operating across a range of scales. 


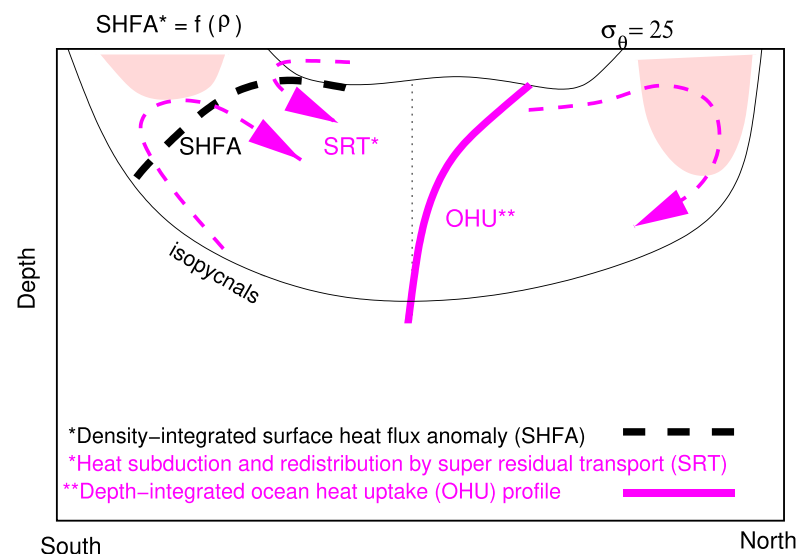

FIG. 10. Schematic view of the OHU process as revealed by the heat budget analyses. Most of the OHU occurs by the advective component of the super residual transport (SRT), which links heat input to different density classes at the surface at mid and high latitudes with $\mathrm{OHU}$ anomalies in the ocean interior, through subduction along isopycnals and heat redistribution from the regions of deep mixing (shaded).

Two complementary approaches are used for the heat budget analysis: a traditional approach that uses horizontal and/or vertical integration of the heat budget components, and an approach that formulates the heat budget within potential density layers (i.e., diapycnal/isopycnal framework).

Using the traditional approach, we find that at statistical steady-state (in the piControl simulation) a leading-order global heat balance in the subsurface upper ocean $(\sim 200$ 2000-m layer) is between the large-scale circulation warming it and mesoscale processes cooling it. This result is consistent with Gregory (2000) and some others (e.g., Griffies et al. 2015; Morrison et al. 2013; Saenko 2006). Parameterized small-scale diapycnal processes do not contribute substantially to the global heat balance in this layer and have a relatively small quantitative spread across the models when compared to the spread in processes operating at larger scales. In general, intermodel spread increases toward the surface for all scales.

In the climate change experiment, the processes representing all scales contribute positively to the subsurface OHU. The contribution from small-scale processes is largest in the upperocean regions poleward of roughly $40^{\circ} \mathrm{S}-40^{\circ} \mathrm{N}$. These regions are where weakening of convective mixing leads to more heat being trapped in the subsurface ocean rather than being ventilated through convection. Below about $300-400 \mathrm{~m}$, OHU is dominated by the superresidual transport (SRT) representing large-scale ocean dynamics combined with all parameterized (in these AOGCMs) mesoscale and submesoscale advective and diffusive eddy effects. Thus, the processes included in SRT not only contribute to the subduction of newly formed water masses (Luyten et al. 1983; Marshall 1997; England and MaierReimer 2001; Dias et al. 2020a), but also control the alongisopycnal penetration of heat anomalies from the mixed layer into the oceanic interior, as described in section $3 \mathrm{~b}$. The contribution of isopycnal diffusion to OHU by SRT is less important than the contribution of the net (resolved plus eddy induced) advection. Overall, there is much better agreement among the AOGCMs in the net global OHU than in the individual scales/processes contributing to it; the same applies to the Atlantic OHU. This behavior implies some degree of compensation between different scales contributing to the global OHU, with the latter tending to self-adjust to the uncertainties in the representation of unresolved ocean physics in AOGCMs. Uncertainties generally increase toward the surface.

While the spatial structure of OHU varies across the models, with the spread being particularly large in the North Atlantic and in the Southern Ocean, the net integrated OHU values simulated by the AOGCMs are remarkably similar. This behavior is despite many differences among the models, including choices made to represent parameterized ocean eddy effects. To put the smallness of the OHU spread into context, we show that the subsurface OHU normalized by the model-mean temperature change in the upper ocean varies much less than does a proxy to OHU efficiency. There are also some common features in the analyzed models, which may have contributed to the small spread in the global OHU. One such feature is that, unlike in some older models (e.g., Wiebe and Weaver 1999), all analyzed models employ neutral physics (Redi 1982; Gent and McWilliams 1990; Gent et al. 1995; Griffies 1998) to represent tracer diffusive mixing and advection by mesoscale eddies. Another common feature is that most of these models impose a rather small (order of $10^{-5} \mathrm{~m}^{2} \mathrm{~s}^{-1}$ ) vertical diffusivity over vast ocean regions in the pycnocline, such as estimated by field measurements (e.g., Ledwell et al. 1993). These common model features thus lead to the interior ocean circulation that tends to follow isopycnals. As a result, the models favor heat uptake that occurs along isopyncals rather than across, with this process contrary to the assumptions of one-dimensional box models of OHU (e.g., Raper et al. 2001).

Regionally, weakening of the large-scale component of the AMOC leads to less heat convergence north of about $40^{\circ} \mathrm{N}$ in the Atlantic and less heat divergence at lower latitudes. As a result of this north-south heat redistribution, the subpolar Atlantic becomes colder, while the rest of the Atlantic becomes warmer, with little overall impact on the net Atlantic Ocean heat content from changes in the large-scale ocean circulation. However, while in the subpolar North Atlantic the cooling induced by changes in the large-scale dynamics is more than offset by subsurface warming due to changes in the parameterized processes (convection and eddy effects), at low latitudes the large-scale heat convergence is not offset by any major process, thereby dominating the local heat content change. In the Southern Ocean, which accounts for about $40 \%$ of the net subsurface OHU on the model mean, the importance of a particular scale strongly depends on latitude, with the OHU south of $50^{\circ} \mathrm{S}$ being mainly due to the parameterized processes.

Using a potential density (diapycnal/isopycnal) framework for the heat budget analysis we find that, at statistical steady state, heat loss at the surface within denser waters is resupplied by small-scale diapycnal mixing and also by the large-scale circulation, particularly at the highest density classes. In the climate change experiment, the potential density framework 
reveals that most of the interior OHU processes are isopycnal in nature, at least outside of the near-surface low-latitude regions. Consequently, we are able to show that most of the global vertical ocean warming profile can be reconstructed by projecting surface heat flux anomalies in the analyzed AOGCMs from potential density space onto the mean depths of the corresponding isopycnals. It can therefore be concluded that heat uptake in the ocean can be broadly explained by heat fluxes into outcropping density layers and near-adiabatic distribution of heat within those layers. This feature, combined with the mostly advective nature of OHU, may have important applications. For example, it supports the construction of simple models of thermosteric sea level rise that are based on the assumptions that 1) the upper layers of the low-latitude ocean are ventilated by the subduction of water at higher latitudes along surfaces of constant density, and 2) heat enters the ocean interior mostly by an advection process rather than by vertical diffusion (Church et al. 1991).

To summarize, our main conclusions are as follows:

1) At steady state, a leading-order global heat balance in the subsurface upper ocean is between the large-scale circulation warming it and mesoscale processes cooling it.

2) The $\mathrm{CO}_{2}$-induced $\mathrm{OHU}$ is dominated by the advective component of the superresidual transport, away from the localized high-latitude regions of strong vertical mixing.

3) The model spread of net OHU is small compared with the spread in components of it, with the ocean warming uncertainties generally increasing toward the surface.

4) There are large uncertainties in the regional $\mathrm{OHC}$ changes, especially in the subpolar North Atlantic, Arctic Ocean, and Southern Ocean.

5) In the Atlantic, most of the OHU is due to the parameterized processes, with changes in the large-scale heat convergence (e.g., due to AMOC weakening) mostly redistributing heat from the north to the south.

6) The dominance of advective heat redistribution in the lowlatitude heat content change is contrary to the diffusive $\mathrm{OHU}$ mechanism assumed by the upwelling-diffusion model.

7) Most of the interior OHU processes are isopycnal in nature, which makes it possible to quite accurately reconstruct much of the global vertical ocean warming profile from the surface heat flux anomalies. This result supports the construction of advective (rather than diffusive) models of OHU and sea level rise.

Acknowledgments. The authors thank Patrick Cummins for providing the observational estimates of vertical heat transport used in Fig. 2 and also Andrew Shao, Michael Winton, and three reviewers for helpful comments. We also thank Abhishek Savita, Helmuth Haak, Bill Hurlin, and Duo Yang for their help and advice with model data. Work by Jonathan Gregory and Matthew Couldrey was supported by Grant NE/R000727/1 from the U.K. Natural Environment Research Council. Fabio Boeira Dias was supported by the Academy of Finland (Project 322432). We are grateful to the climate modeling groups for producing and making available their model output, the Earth System Grid Federation (ESGF) for archiving the data and the multiple funding agencies who support CMIP5, CMIP6, and ESGF. The CMIP6 data used in the study are available from https://esgf-data.dkrz.de/projects/cmip6-dkrz/.

\section{APPENDIX A}

\section{Projection of the Eulerian Budgets of Heat and Salt onto Density Surfaces}

Here we present the approach we use for projecting the Eulerian heat budget terms onto the position of density surfaces. We also show how this approach can be applied to the Eulerian salinity budget and draw some parallels with the WMT framework described in Groeskamp et al. (2019).

Consider the heat budget in the following form [cf. Eq. (51) in Groeskamp et al. 2019]:

$$
\begin{aligned}
C_{p} \rho \partial_{t} \Theta & =-C_{p} \rho \mathbf{u}^{\dagger} \cdot \nabla \Theta-\nabla \cdot \mathbf{J}_{Q}-\nabla \cdot \mathbf{J}_{Q}^{\mathrm{swr}} \\
& +\left[F_{Q}+C_{p} Q_{m}\left(\Theta_{m}-\Theta\right)\right] \delta(z-\eta),
\end{aligned}
$$

where $\Theta$ is the Conservative Temperature and $\nabla \Theta$ is its gradient, $\mathbf{u}^{\dagger}$ is the sum of resolved (u) and eddy-induced $\left(\mathbf{u}^{*}\right)$ velocities in the analyzed models; that is, $\mathbf{u}^{\dagger}=\mathbf{u}+\mathbf{u}^{*}$. Other terms are as follows: $-\nabla \cdot \mathbf{J}_{Q}$ is heat convergence due to interior mixing processes; $-\nabla$. $\mathbf{J}_{Q}^{\text {swr }}$ is heat convergence due to penetrative shortwave radiation; $F_{Q}$ represents heat fluxes at the ocean surface $[z=\eta(x, y, t)]$, with the penetrated shortwave radiation excluded; $C_{p} Q_{m}\left(\Theta_{m}-\Theta\right)$ accounts for the heat content of mass transferred through the surface, with $\Theta_{m}$ being the Conservative Temperature of the corresponding mass flux $Q_{m}$ that can be associated with, for example, precipitation minus evaporation and river runoff. Some other terms, such as the geothermal heat flux at the ocean bottom, can also be included in Eq. (A1). Integrating Eq. (A1) over all ocean regions with densities larger than any given density $\rho$ and averaging in time (denoted with overbar) gives

$$
\begin{aligned}
& \underbrace{\overline{\iint_{\rho^{\prime}(x, y, z, t) \geq \rho} C_{p} \rho \partial_{t} \Theta d V}}_{\text {All scales }}=\underbrace{\iiint_{\rho^{\prime}(x, y, z, t) \geq \rho}-C_{p} \rho \mathbf{u} \cdot \nabla \Theta d V}_{\text {Large }}+\underbrace{\iiint_{\rho^{\prime}(x, y, z, t) \geq \rho}-C_{p} \rho \mathbf{u}^{*} \cdot \nabla \Theta d V}_{\text {Meso and Small }}+\overline{\iiint_{\rho^{\prime}(x, y, z, t) \geq \rho}-\nabla \cdot \mathbf{J}_{Q} d V}
\end{aligned}
$$

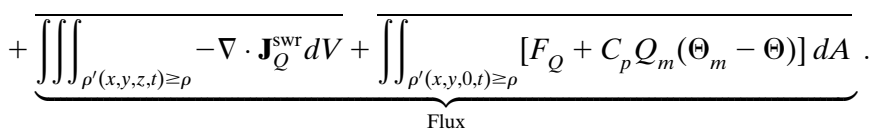


This equation [cf. Eq. (11)] presents the essence of our approach for projecting the Eulerian heat budget onto the position of density surfaces, followed by averaging in time. Note that the terms containing $\mathbf{u}$ and $\mathbf{u}^{*}$ represent the diapycnal transports associated with, respectively, the resolved and eddy-induced heat advection across density surfaces that occurs in the presence of mixing and heat input at the surface. In the heat budget projection onto density surfaces presented in section $3 b$, focused mostly on the upper $2 \mathrm{~km}$ of the ocean, we use $\sigma_{\theta}$; a similar calculation can be applied using other types of density.

Consider now the budget of salinity $S$ [cf. Eq. (50) in Groeskamp et al. 2019]:

$$
\rho \partial_{t} S=-\rho \mathbf{u}^{\dagger} \cdot \nabla S-\nabla \cdot \mathbf{J}_{S}+\left[F_{S}+Q_{m}\left(S_{m}-S\right)\right] \delta(z-\eta),
$$

where $-\nabla \cdot \mathbf{J}_{S}$ represents the interior mixing, $F_{S}$ is the exchange of salt and freshwater across the surface, and $S_{m}-S$ is the difference between the salinity in the transferred mass and the sea surface salinity. By applying the operator $\iiint_{\rho^{\prime}(x, y, z, t) \geq \rho}() d$.$V to$ each its term and averaging in time, a projection of the Eulerian salt budget onto density surfaces can be constructed, similar to Eq. (A2).

We note that the described projection of the Eulerian heat and salinity budgets onto density surfaces is more straightforward than the isopycnal tracer budget discussed by Groeskamp et al. (2019). The latter has many intricacies, discussion of which is beyond our scope. Instead, we draw only some parallels with the WMT framework presented in Groeskamp et al. (2019). In particular, Groeskamp et al. (2019) use the material evolution of Conservative Temperature

$$
C_{p} \rho \dot{\Theta}=-\nabla \cdot \mathbf{J}_{Q}-\nabla \cdot \mathbf{J}_{Q}^{\mathrm{swr}}+\left[F_{Q}+C_{p} Q_{m}\left(\Theta_{m}-\Theta\right)\right] \delta(z-\eta),
$$

where in our case $\dot{\Theta}=\partial_{t} \Theta+\mathbf{u}^{\dagger} \cdot \nabla \Theta$, and the material evolution of salinity

$$
\rho \dot{S}=-\nabla \cdot \mathbf{J}_{S}+\left[F_{S}+Q_{m}\left(S_{m}-S\right)\right] \delta(z-\eta),
$$

where $\dot{S}=\partial_{t} S+\mathbf{u}^{\dagger} \cdot \nabla S$. By multiplying Eq. (A4) by $-\alpha / C_{p}$, where $\alpha$ is the thermal expansion coefficient, and Eq. (A5) by the haline contraction coefficient $\beta$, and adding the results, one can arrive at the equation for material evolution of locally referenced potential density; that is, similar to Eq. (A4) in Groeskamp et al. (2019). The latter, upon conversion to the material evolution of neutral density $\gamma$ [Eq. (12) in Groeskamp et al. 2019] and applying the operator $(\partial / \partial \gamma) \iiint_{\gamma^{\prime} \leq \gamma}() d$.$V , forms$ the core of the WMT framework. For example, the term $\left(\alpha / C_{p}\right) \nabla \cdot \mathbf{J}_{Q}-\beta \nabla \cdot \mathbf{J}_{S}$ in the resulting WMT equation leads to the transformation associated with mixing at different scales as well as to transformation arising due to nonlinearities in the equation of state (i.e., cabbeling and thermobaricity). In turn, the terms $\left[-\left(\alpha / C_{p}\right) F_{Q}+\beta F_{S}\right] \delta(z-\eta)$ and $\left[-\alpha Q_{m}\left(\Theta_{m}-\Theta\right)+\right.$ $\left.\beta Q_{m}\left(S_{m}-S\right)\right] \delta(z-\eta)$ lead to WMT associated, respectively, with the surface flux of density and the density source due to mass influx at the surface.

\section{APPENDIX B}

\section{Covariance Between the Upper-Ocean Warming to Subsurface OHU}

Consider the evolution of global-mean profile of ocean temperature anomaly, $\theta(z, t)$, as defined relative to some unforced control state. For example, in our study $\theta(z, t)$ is the horizontally averaged vertical profile of temperature in the 1 pctCO2 simulation relative to the piControl. The evolution of $\theta(z, t)$ can be described by the following equation

$$
C \partial_{t} \theta=\partial_{z} \mathscr{F},
$$

where $C$ is the volumetric heat capacity of seawater, $\mathscr{F}$ is the forcing due to global-mean air-sea heat flux anomaly as well as all vertical heat transport processes, and $\theta(z, t=0)=0$. Integrating Eq. (B1) vertically from the surface to some depth and then averaging over time $0 \leq t \leq \tau$, with $\theta(t=\tau)=\theta_{\tau}$, gives

$$
\Delta \mathscr{T}(z)=\mathscr{F}_{0}-\mathrm{OHU}(z)
$$

where $\Delta \mathscr{T}(z)=C / \tau \int_{z}^{0} \theta_{\tau} d z$ is the heat convergence in the layer above $z$, which is proportional to the layer's temperature change, $\mathscr{F}_{0}$ ( $>0$ when comparing 1 pctCO 2 relative to piControl) is the time-mean air-sea heat flux anomaly and $\mathrm{OHU}(z) \equiv \mathscr{F}(z)$ is the time-mean heat uptake by the ocean below $z$ (assuming no geothermal or Joule heating).

Now introduce a model ensemble mean by $\langle a\rangle$ and recall the definitions of variance, $\operatorname{var}(a) \equiv\left\langle(a-\langle a\rangle)^{2}\right\rangle$ and covariance, $\operatorname{cov}(a, b) \equiv\langle(a-\langle a\rangle)(b-\langle b\rangle)\rangle$. The heat budget Eq. (B2) thus gives

$$
\operatorname{cov}(\Delta \mathscr{T}, \mathrm{OHU})=\operatorname{cov}\left(\mathscr{F}_{0}, \mathrm{OHU}\right)-\operatorname{var}(\mathrm{OHU}) .
$$

Hence, we see that the covariance between the heat convergence in the upper ocean (or upper-ocean temperature change) and heat flux anomaly into the ocean below $[\mathrm{OHU}(z)]$ increases with the covariance between the surface heat flux anomaly and $\mathrm{OHU}(z)$ and decreases with the variance of $\mathrm{OHU}(z)$.

\section{REFERENCES}

Bi, D., and Coauthors, 2020: Configuration and spin-up of ACCESS-CM2, the new generation Australian community climate and Earth system simulator coupled model. J. South. Hemisphere Earth Syst. Sci., 70, 225-251, https://doi.org/ 10.1071/ES19040.

Boucher, O., and Coauthors, 2020: Presentation and evaluation of the IPSL-CM6A-LR climate model. J. Adv. Model. Earth Syst., 12, e2019MS002010, https://doi.org/10.1029/2019MS002010.

Church, J. A., J. S. Godfrey, D. R. Jackett, and T. J. McDougall, 1991: A model of sea level rise caused by ocean thermal expansion. J. Climate, 4, 438-456, https://doi.org/10.1175/15200442(1991)004<0438:AMOSLR>2.0.CO;2.

, N. White, C. Domingues, D. Monselesan, and E. Miles, 2013: Sea-level change and ocean heat-content change. Ocean Circulation and Climate: A 21st Century Perspective, 2nd ed. G. Siedler et al., Eds., International Geophysics Series, Vol. 103, Academic Press, 697-726.

Couldrey, M. P., and Coauthors, 2021: What causes the spread of model projections of ocean dynamic sea level change in 
response to greenhouse gas forcing? Climate Dyn., 56, 155187, https://doi.org/10.1007/s00382-020-05471-4.

Cummins, P. F., D. Masson, and O. A. Saenko, 2016: Vertical heat flux in the ocean: Estimates from observations and from a coupled general circulation model. J. Geophys. Res. Oceans, 121, 3790-3802, https://doi.org/10.1002/2016JC011647.

Danabasoglu, G., S. C. Bates, B. P. Briegleb, S. R. Jayne, M. Jochum, W. G. Large, S. Peacock, and S. G. Yeager, 2012: The CCSM4 ocean component. J. Climate, 25, 1361-1389, https://doi.org/10.1175/JCLI-D-11-00091.1.

Dias, F. B., C. M. Domingues, S. Marsland, S. Griffies, S. Rintoul, R. Matear, and R. Fiedler, 2020a: On the superposition of mean advective and eddy-induced transports in global ocean heat and salt budgets. J. Climate, 33, 1121-1140, https:// doi.org/10.1175/JCLI-D-19-0418.1.

— changing ocean circulation processes. J. Climate, 33, 90659082, https://doi.org/10.1175/JCLI-D-19-1016.1.

Dunne, J. P., and Coauthors, 2012: GFDL's ESM2 global coupled climate-carbon Earth system models. Part I: Physical formulation and baseline simulation characteristics. J. Climate, 25, 6646-6665, https://doi.org/10.1175/JCLI-D-11-00560.1.

England, M., and E. Maier-Reimer, 2001: Using chemical tracers to assess ocean models. Rev. Geophys., 39, 29-70, https://doi.org/ 10.1029/1998RG000043.

Exarchou, E., T. Kuhlbrodt, J. M. Gregory, and R. S. Smith, 2015: Ocean heat uptake processes: A model intercomparison. J. Climate, 28, 887-908, https://doi.org/10.1175/JCLI-D-14-00235.1.

Fox-Kemper, B., G. Danabasoglu, R. Ferrari, S. M. Griffies, R. W. Hallberg, M. Holland, S. Peacock, and B. Samuels, 2011: Parameterization of mixed layer eddies. III: Implementation and impact on global ocean climate simulations. Ocean Modell., 39, 61-78, https://doi.org/10.1016/j.ocemod.2010.09.002.

Gent, P. R., and J. C. McWilliams, 1990: Isopycnal mixing in ocean general circulation models. J. Phys. Oceanogr., 20, 150-155, https:// doi.org/10.1175/1520-0485(1990)020<0150:IMIOCM>2.0.CO;2.

_ Parameterizing eddy-induced tracer transports in ocean circulation models. J. Phys. Oceanogr., 25, 463-474, https://doi.org/ 10.1175/1520-0485(1995)025<0463:PEITTI >2.0.CO;2.

Gnanadesikan, A., R. D. Slater, P. S. Swathi, and G. K. Vallis, 2005: The energetics of ocean heat transport. J. Climate, 18, 2604 2616, https://doi.org/10.1175/JCLI3436.1.

Gordon, C., C. Cooper, C. A. Senior, H. T. Banks, J. M. Gregory, T. C. Johns, J. F. B. Mitchell, and R. A. Wood, 2000: The simulation of SST, sea ice extents and ocean heat transports in a version of the Hadley Centre coupled model without flux adjustments. Climate Dyn., 16, 147-168, https://doi.org/ 10.1007/s003820050010.

Gregory, J. M., 2000: Vertical heat transports in the ocean and their effect on time-dependent climate change. Climate Dyn., 16, 501-515, https://doi.org/10.1007/s003820000059.

_- and R. Tailleux, 2011: Kinetic energy analysis of the response of the Atlantic meridional overturning circulation to $\mathrm{CO}_{2}$ forced climate change. Climate Dyn., 37, 893-914, https:// doi.org/10.1007/s00382-010-0847-6.

—_, and Coauthors, 2016: The Flux-Anomaly-Forced Model Intercomparison Project (FAFMIP) contribution to CMIP6: Investigation of sea-level and ocean climate change in response to $\mathrm{CO}_{2}$ forcing. Geosci. Model Dev., 9, 3993-4017, https://doi.org/10.5194/gmd-9-3993-2016.
Griffies, S. M., 1998: The Gent-McWilliams skew flux. J. Phys. Oceanogr., 28, 831-841, https://doi.org/10.1175/1520-0485(1998) $028<0831$ :TGMSF $>2.0$. CO 2 .

_- A. Gnanadesikan, R. C. Pacanowski, V. Larichev, J. K. Dukowicz, and R. D. Smith, 1998: Isoneutral diffusion in a $z$-coordinate ocean model. J. Phys. Oceanogr., 28, 805-830, https://doi.org/10.1175/15200485(1998)028<0805:IDIAZC $>2.0 . C O ; 2$.

— , and Coauthors, 2015: Impacts on ocean heat from transient mesoscale eddies in a hierarchy of climate models. J. Climate, 28, 952-977, https://doi.org/10.1175/JCLI-D-14-00353.1.

—, and Coauthors, 2016: OMIP contribution to CMIP6: Experimental and diagnostic protocol for the physical component of the Ocean Model Intercomparison Project. Geosci. Model Dev., 9, 3231-3296, https://doi.org/10.5194/ gmd-9-3231-2016.

Groeskamp, S., J. D. Zika, T. J. McDougall, B. M. Sloyan, and F. Laliberté, 2014: The representation of ocean circulation and variability in thermodynamic coordinates. J. Phys. Oceanogr., 44, 1735-1750, https://doi.org/10.1175/JPO-D-13-0213.1.

, S. M. Griffies, D. Iudicone, R. Marsh, A. J. G. Nurser, and J. D. Zika, 2019: The water mass transformation framework for ocean physics and biogeochemistry. Annu. Rev. Mar. Sci., 11, 271-305, https://doi.org/10.1146/annurev-marine-010318095421.

Gutjahr, O., D. Putrasahan, K. Lohmann, J. H. Jungclaus, J.-S. von Storch, N. Brüggemann, H. Haak, and A. Stössel, 2019: Max Planck Institute Earth System Model (MPI-ESM1.2) for the High-Resolution Model Intercomparison Project (HighResMIP). Geosci. Model Dev., 12, 3241-3281, https://doi.org/10.5194/gmd-123241-2019.

Holmes, R. M., J. D. Zika, and M. H. England, 2019: Diathermal heat transport in a global ocean model. J. Phys. Oceanogr., 49, 141-161, https://doi.org/10.1175/JPO-D-18-0098.1.

Johns, T. C., and Coauthors, 2006: The new Hadley Centre climate model (HadGEM1): Evaluation of coupled simulations. J. Climate, 19, 1327-1353, https://doi.org/10.1175/JCLI3712.1.

Khatiwala, S., and M. Visbeck, 2000: An estimate of the eddyinduced circulation in the Labrador Sea. Geophys. Res. Lett., 27, 2277-2280, https://doi.org/10.1029/1999GL011073.

Kuhlbrodt, T., and J. M. Gregory, 2012: Ocean heat uptake and its consequences for the magnitude of sea level rise and climate change. Geophys. Res. Lett., 39, L18608, https://doi.org/ $10.1029 / 2012$ GL052952.

- , - and L. C. Shaffrey, 2015: A process-based analysis of ocean heat uptake in an AOGCM with an eddy-permitting ocean component. Climate Dyn., 45, 3205-3226, https:// doi.org/10.1007/s00382-015-2534-0.

— HadGEM3 GC3.1: Development and evaluation for global climate. J. Adv. Model. Earth Syst., 10, 2865-2888, https:// doi.org/10.1029/2018MS001370.

Ledwell, J. R., A. J. Watson, and C. S. Law, 1993: Evidence for slow mixing across the pycnocline from an open-ocean tracer-release experiment. Nature, 364, 701-703, https://doi.org/10.1038/364701a0.

Levitus, S., and Coauthors, 2012: World ocean heat content and thermosteric sea level change (0-2000 m), 1955-2010. Geophys. Res. Lett., 39, L10603, https://doi.org/10.1029/2012GL051106.

Luyten, J. R., J. Pedlosky, and H. Stommel, 1983: The ventilated thermocline. J. Phys. Oceanogr., 13, 292-309, https://doi.org/ 10.1175/1520-0485(1983)013<0292:TVT>2.0.CO;2.

Marshall, D. P., 1997: Subduction of water masses in an eddying ocean. J. Mar. Res., 55, 201-222, https://doi.org/10.1357/ 0022240973224373 . 
Morrison, A. K., O. A. Saenko, A. M. Hogg, and P. Spence, 2013: The role of vertical eddy flux in Southern Ocean heat uptake. Geophys. Res. Lett., 40, 5445-5450, https://doi.org/10.1002/ 2013GL057706.

Munk, W. H., 1966: Abyssal recipes. Deep-Sea Res. Oceanogr. Abstr., 13, 707-730, https://doi.org/10.1016/0011-7471(66) 90602-4.

—_, and C. Wunsch, 1998: Abyssal recipes II: Energetics of tidal and wind mixing. Deep-Sea Res., 45, 1977-2010, https:// doi.org/10.1016/S0967-0637(98)00070-3.

Naveira Garabato, A. C., L. Jullion, D. P. Stevens, K. J. Heywood, and B. A. King, 2009: Variability of Subantarctic Mode Water and Antarctic Intermediate Water in the Drake Passage during the late-twentieth and early-twenty-first centuries. J. Climate, 22, 3661-3688, https://doi.org/10.1175/2009JCLI2621.1.

Otto, A., and Coauthors, 2013: Energy budget constraints on climate response. Nat. Geosci., 6, 415-416, https://doi.org/ 10.1038/ngeo1836.

Raper, S. C. B., J. M. Gregory, and T. J. Osborn, 2001: Use of an upwelling-diffusion energy balance climate model to simulate and diagnose A/OGCM results. Climate Dyn., 17, 601-613, https://doi.org/10.1007/PL00007931.

Redi, M. H., 1982: Oceanic isopycnal mixing by coordinate rotation. J. Phys. Oceanogr., 12, 1154-1158, https://doi.org/10. 1175/1520-0485(1982)012<1154:OIMBCR > 2.0.CO;2.

Saba, V. S., and Coauthors, 2016: Enhanced warming of the northwest Atlantic Ocean under climate change. J. Geophys. Res. Oceans, 121, 118-132, https://doi.org/10.1002/2015JC011346.

Saenko, O. A., 2006: The effect of localized mixing on the ocean circulation and time-dependent climate change. J. Phys. Oceanogr., 36, 140-160, https://doi.org/10.1175/JPO2839.1.
_ J. C. Fyfe, and M. H. England, 2005: On the response of the oceanic wind-driven circulation to atmospheric $\mathrm{CO}_{2}$ increase. Climate Dyn., 25, 415-426, https://doi.org/10.1007/s00382-005-0032-5.

Swart, N. C., and Coauthors, 2019: The Canadian Earth System Model version 5 (CanESM5.0.3). Geosci. Model Dev. Discuss., 12, 4823-4873, https://doi.org/10.5194/gmd-12-4823-2019.

Tomczak, M., and J. S. Godfrey, 1994: Regional Oceanography: An Introduction. Pergamon, $422 \mathrm{pp}$.

Walin, G., 1982: On the relation between sea-surface heat flow and thermal circulation in the ocean. Tellus, 34, 187-195, https:// doi.org/10.1111/j.2153-3490.1982.tb01806.x.

Wiebe, E., and A. Weaver, 1999: On the sensitivity of global warming experiments to the parametrisation of sub-grid scale ocean mixing. Climate Dyn., 15, 875-893, https://doi.org/ $10.1007 / \mathrm{s} 003820050319$.

Yang, D., and O. A. Saenko, 2012: Ocean heat transport and its projected change in CanESM2. J. Climate, 25, 8148-8163, https://doi.org/10.1175/JCLI-D-11-00715.1.

Yin, J., S. M. Griffies, and R. J. Stouffer, 2010: Spatial variability of sea level rise in twenty-first century projections. J. Climate, $\mathbf{2 3}$, 4585-4607, https://doi.org/10.1175/2010JCLI3533.1.

Yukimoto, S., and Coauthors, 2019: The Meteorological Research Institute Earth System Model version 2.0, MRI-ESM2.0: Description and basic evaluation of the physical component. J. Meteor. Soc. Japan, 97, 931-965, https://doi.org/10.2151/ jmsj.2019-051.

Zanna, S., L. Khatiwala, J. Gregory, J. Ison, and P. Heimbach, 2019: Global reconstruction of historical ocean heat storage and transport. Proc. Natl. Acad. Sci. USA, 116, 1126-1131, https://doi.org/10.1073/pnas.1808838115. 